TRANSACTIONS OF THE

AMERICAN MATHEMATICAL SOCIETY

Volume 364, Number 8, August 2012, Pages 4323-4368

S 0002-9947(2012)05585-1

Article electronically published on March 20, 2012

\title{
AN ASYMPTOTIC FORMULA FOR THE NUMBER OF NON-NEGATIVE INTEGER MATRICES WITH PRESCRIBED ROW AND COLUMN SUMS
}

\author{
ALEXANDER BARVINOK AND J. A. HARTIGAN
}

\begin{abstract}
We count $m \times n$ non-negative integer matrices (contingency tables) with prescribed row and column sums (margins). For a wide class of smooth margins we establish a computationally efficient asymptotic formula approximating the number of matrices within a relative error which approaches 0 as $m$ and $n$ grow.
\end{abstract}

\section{Introduction AND MAin RESUlts}

Let $R=\left(r_{1}, \ldots, r_{m}\right)$ and $C=\left(c_{1}, \ldots, c_{n}\right)$ be positive integer vectors such that

$$
r_{1}+\ldots+r_{m}=c_{1}+\ldots+c_{n}=N .
$$

We are interested in the number \#(R,C) of $m \times n$ non-negative integer matrices $D=\left(d_{i j}\right)$ with row sums $R$ and column sums $C$. Such matrices $D$ are often called contingency tables with margins $(R, C)$. The problem of computing or estimating $\#(R, C)$ efficiently has attracted considerable attention; see, for example, [8], 9], [20, 15], 16, 18, 23, 12, [11, 10, [19, 7], 25] and [3.

Asymptotic formulas for numbers \# $(R, C)$ as $m$ and $n$ grow are known in sparse cases, where the average entry $N / m n$ of the matrix goes to 0 , see [8], [9], [19] and in the case when all row and all column sums are equal, $r_{1}=\ldots=r_{m}$ and $c_{1}=\ldots=c_{n}$, [10. In [3] an asymptotic formula for $\log \#(R, C)$ is established under quite general circumstances.

In this paper, we prove an asymptotic formula for $\#(R, C)$ for a reasonably wide class of smooth margins $(R, C)$. In 6 we apply a similar approach to find an asymptotic formula for the number of matrices (binary contingency tables) with row sums $R$, column sums $C$ and $0-1$ entries as well as to find an asymptotic formula for the number of graphs with prescribed degrees of vertices.

1.1. The typical matrix and smooth margins. The typical matrix was introduced in [3] and various versions of smoothness for margins were introduced in [7] and in 4. The function

$$
g(x)=(x+1) \ln (x+1)-x \ln x \quad \text { for } \quad x \geq 0
$$

Received by the editors April 5, 2010 and, in revised form, March 11, 2011, and March 18, 2011.

2010 Mathematics Subject Classification. Primary 05A16, 52B55, 52C07, $60 \mathrm{~F} 05$.

The research of the first author was partially supported by NSF Grant DMS 0856640 and a United States-Israel BSF grant 2006377.

(C)2012 American Mathematical Society Reverts to public domain 28 years from publication 
plays the crucial role. It is easy to see that $g$ is increasing and concave with $g(0)=0$. For an $m \times n$ non-negative matrix $X=\left(x_{j k}\right)$ we define

$$
g(X)=\sum_{\substack{1 \leq j \leq m \\ 1 \leq k \leq n}} g\left(x_{j k}\right)=\sum_{\substack{1 \leq j \leq m \\ 1 \leq k \leq n}}\left(\left(x_{j k}+1\right) \ln \left(x_{j k}+1\right)-x_{j k} \ln x_{j k}\right) .
$$

Given margins $(R, C)$, let $P(R, C)$ be the polytope of all real non-negative $m \times n$ matrices $X=\left(x_{j k}\right)$ with row sums $R$ and column sums $C$, also known as the transportation polytope. We consider the following optimization problem:

$$
\text { Find } \max _{X \in P(R, C)} g(X) \text {. }
$$

Since $g$ is strictly concave, the maximum is attained at a unique matrix $Z=\left(\zeta_{j k}\right)$, which we call the typical matrix with margins $(R, C)$. One can show that $\zeta_{j k}>0$ for all $j$ and $k$; see [7] and [4. In [4] it is shown that a random contingency table, sampled from the uniform distribution on the set of all non-negative integer matrices with row sums $R$ and column sums $C$ is, in some rigorously defined sense, likely to be close to the typical matrix $Z$. In [5] we give the following probabilistic interpretation of $Z$. Let us consider the family of all probability distributions on the set $\mathbb{Z}_{+}^{m \times n}$ of all non-negative $m \times n$ integer matrices with the expectations in the affine subspace $\mathcal{A}(R, C)$ of the $m \times n$ matrices with row sums $R$ and column sums $C$. In this family there is a unique distribution of the maximum entropy and $Z$ turns out to be the expectation of that distribution. The maximum entropy distribution is necessarily a distribution on $\mathbb{Z}_{+}^{m \times n}$ with independent geometrically distributed coordinates, which, conditioned on $\mathcal{A}(R, C)$, results in the uniform distribution on the set of contingency tables with margins $(R, C)$. Function $g(X)$ turns out to be the entropy of the multivariate geometric distribution on $\mathbb{Z}_{+}^{m \times n}$ with the expectation $X$.

Let us fix a number $0<\delta<1$. We say that margins $(R, C)$ are $\delta$-smooth provided the following conditions (1.2) $-(1.4)$ are satisfied:

$$
m \geq \delta n \text { and } n \geq \delta m
$$

so the dimensions of the matrix are of the same order;

$$
\delta \tau \leq \zeta_{j k} \leq \tau \text { for all } j \text { and } k,
$$

for some $\tau$ such that

$$
\tau \geq \delta
$$

We note that $\delta$-smooth margins are also $\delta^{\prime}$-smooth for any $0<\delta^{\prime}<\delta$.

Condition (1.3) requires that the entries of the typical matrix are of the same order and it plays a crucial role in our proofs. Often, one can show that margins are smooth by predicting what the solution to the optimization problem (1.1) will look like. For example, if all row sums $r_{j}$ are equal, symmetry requires that we have $\zeta_{j k}=c_{k} / m$ for all $j$ and $k$, so the entries of the typical matrix are of the same order provided the column sums $c_{k}$ are of the same order. On the other hand, (1.3) is violated in some curious cases. For example, if $m=n$ and $r_{1}=\ldots=r_{n-1}=c_{1}=$ $\ldots=c_{n-1}=n$ while $r_{n}=c_{n}=3 n$, the entry $\zeta_{n n}$ of the typical matrix is linear in $n$, namely $\zeta_{n n}>0.58 n$, while all other entries of $Z$ remain bounded by a constant; see 4. If we change $r_{n}$ and $c_{n}$ to $2 n$, the entry $\zeta_{n n}$ becomes bounded by a constant as 
well. One may wonder (this question is inspired by a conversation with B. McKay) if the smoothness condition (1.3) is indeed necessary for the number of tables $\#(R, C)$ to be expressible by a formula which varies "smoothly" as the margins $R$ and $C$ vary, such as the formula in Theorem 1.1 below. In particular, can there be a sudden jump in the number of tables with $m=n, r_{1}=\ldots=r_{n-1}=c_{1}=\ldots=c_{n-1}=n$ when $r_{n}=c_{n}$ crosses a certain threshold between $2 n$ and $3 n$ ?

In [7] it is proven that if the ratio of the maximum row sum $r_{+}=\max _{j} r_{j}$ to the minimum row sum $r_{-}=\min _{j} r_{j}$ and the ratio of the maximum column sum $c_{+}=\max _{k} c_{k}$ to the minimum column sum $c_{-}=\min _{k} c_{k}$ do not exceed a number $\beta<(1+\sqrt{5}) / 2 \approx 1.618$, then (1.3) is satisfied with some $\delta=\delta(\beta)>0$. The bound $(1+\sqrt{5}) / 2$ is not optimal, apparently it can be increased to 2 ; see [22]. It looks plausible that if the margins are of the same order and sufficiently generic, then the entries of the typical table are of the same order as well.

The lower bound in (1.4) requires that the density $N / m n$ of the margins, that is the average entry of the matrix, remains bounded away from 0 . This is unavoidable as our asymptotic formula does not hold for sparse cases where $N / m n \longrightarrow 0$; see [19].

We proceed to define various objects needed to state our asymptotic formula.

1.2. Quadratic form $q$ and related quantities. Let $Z=\left(\zeta_{j k}\right)$ be the typical matrix defined in Section 1.1. We consider the following quadratic form $q: \mathbb{R}^{m+n} \longrightarrow \mathbb{R}:$

$$
\begin{aligned}
& q(s, t)=\frac{1}{2} \sum_{\substack{1 \leq j \leq m \\
1 \leq k \leq n}}\left(\zeta_{j k}^{2}+\zeta_{j k}\right)\left(s_{j}+t_{k}\right)^{2} \quad \text { where } \\
& \qquad s=\left(s_{1}, \ldots, s_{m}\right) \text { and } t=\left(t_{1}, \ldots, t_{n}\right) .
\end{aligned}
$$

Thus $q$ is a positive semidefinite quadratic form. It is easy to see that the null-space of $q$ is spanned by vector

$$
u=(\underbrace{1, \ldots, 1}_{m \text { times }} ; \underbrace{-1, \ldots,-1}_{n \text { times }}) .
$$

Let $H=u^{\perp}, H \subset \mathbb{R}^{m+n}$ be the orthogonal complement to $u$. Then the restriction $q \mid H$ is a positive definite quadratic form and hence we can define its determinant $\operatorname{det} q \mid H$ that is the product of the non-zero eigenvalues of $q$. Let us define polynomials $f, h: \mathbb{R}^{m+n} \longrightarrow \mathbb{R}$ by

$$
\begin{aligned}
& f(s, t)=\frac{1}{6} \sum_{\substack{1 \leq j \leq m \\
1 \leq k \leq n}} \zeta_{j k}\left(\zeta_{j k}+1\right)\left(2 \zeta_{j k}+1\right)\left(s_{j}+t_{k}\right)^{3} \\
& \text { and } \\
& h(s, t)=\frac{1}{24} \sum_{\substack{1 \leq j \leq m \\
1 \leq k \leq n}} \zeta_{j k}\left(\zeta_{j k}+1\right)\left(6 \zeta_{j k}^{2}+6 \zeta_{j k}+1\right)\left(s_{j}+t_{k}\right)^{4} .
\end{aligned}
$$

We consider the Gaussian probability measure on $H$ with the density proportional to $e^{-q}$ and define

$$
\mu=\mathbf{E} f^{2} \quad \text { and } \quad \nu=\mathbf{E} h .
$$

Now we have all the ingredients to state our asymptotic formula for $\#(R, C)$. 
Theorem 1.1. Let us fix $0<\delta<1$. Let $(R, C)$ be $\delta$-smooth margins, let the function $g$ and the typical matrix $Z$ be as defined in Section 1.1 and let the quadratic form $q$ and values of $\mu$ and $\nu$ be as defined in Section 1.2, Then the value of

$$
\frac{e^{g(Z)} \sqrt{m+n}}{(4 \pi)^{(m+n-1) / 2} \sqrt{\operatorname{det} q \mid H}} \exp \left\{-\frac{\mu}{2}+\nu\right\}
$$

approximates $\#(R, C)$ within a relative error which approaches 0 as $m, n \longrightarrow+\infty$. More precisely, for any $0<\epsilon \leq 1 / 2$ the above expression approximates $\#(R, C)$ within relative error $\epsilon$ provided

$$
m+n \geq\left(\frac{1}{\epsilon}\right)^{\gamma(\delta)}
$$

for some $\gamma(\delta)>0$.

In [10] Canfield and McKay obtain an asymptotic formula for $\#(R, C)$ in the particular case of all row sums being equal and all column sums being equal. One can show that our formula indeed becomes the asymptotic formula of [10] when $r_{1}=\ldots=r_{m}$ and $c_{1}=\ldots=c_{n}$. In [3] it is proven that the value $g(Z)$ provides an asymptotic approximation to $\ln \#(R, C)$ for a rather wide class of margins (essentially, we need only the density $N / m n$ to be bounded away from 0 but do not need a subtler condition (1.3) of smoothness). The first part

$$
\frac{e^{g(Z)} \sqrt{m+n}}{(4 \pi)^{(m+n-1) / 2} \sqrt{\operatorname{det} q \mid H}}
$$

of the formula is called the "Gaussian approximation" in [5]. It has the following intuitive explanation. Let us consider a random matrix $X$ with the multivariate geometric distribution on the set $\mathbb{Z}_{+}^{m \times n}$ of all non-negative integer matrices such that $\mathbf{E} X=Z$, where $Z$ is the typical matrix with margins $(R, C)$. It follows from the results of [5] that the distribution of $X$ conditioned on the affine subspace $\mathcal{A}=\mathcal{A}(R, C)$ of matrices with row sums $R$ and column sums $C$ is uniform with the probability mass function of $e^{-g(Z)}$ for every non-negative integer matrix in $\mathcal{A}$. Therefore,

$$
\#(R, C)=e^{g(Z)} \mathbf{P}\{X \in \mathcal{A}\} .
$$

Let $Y \in \mathbb{R}^{m+n}$ be a random vector obtained by computing $m$ row sums and $n$ column sums of $X$. Then $\mathbf{E} Y=(R, C)$ and

$$
\mathbf{P}\{X \in \mathcal{A}\}=\mathbf{P}\{Y=(R, C)\} .
$$

We obtain (1.7) if we assume in the spirit of the Local Central Limit Theorem that the distribution of $Y$ in the vicinity of $\mathbf{E} Y$ is close to the $(m+n-1)$-dimensional Gaussian distribution (we lose one dimension since the row and column sums of a matrix are bound by one linear relation: the sum of all row sums is equal to the sum of all column sums). This assumption is not implausible since the coordinates of $Y$ are obtained by summing up of a number of independent entries of $X$.

The correction factor

$$
\exp \left\{-\frac{\mu}{2}+\nu\right\}
$$

is, essentially, the Edgeworth correction in the Central Limit Theorem. In the course of the proof of Theorem 1.1 we establish a two-sided bound

$$
\gamma_{1}(\delta) \leq \exp \left\{-\frac{\mu}{2}+\nu\right\} \leq \gamma_{2}(\delta)
$$


for some constants $\gamma_{1}(\delta), \gamma_{2}(\delta)>0$ as long as the margins $(R, C)$ remain $\delta$-smooth.

De Loera 13, 14] ran a range of numerical experiments which seem to demonstrate that already the Gaussian approximation (1.7) works reasonably well for contingency tables. Thus for $R=(220,215,93,64)$ and $C=(108,286,71,127)$ formula (1.7) approximates \# $(R, C)$ within a relative error of about $6 \%$, for $R=C=$ $(300,300,300,300)$ the error is about $12 \%$ while for $R=(65205,189726,233525$, $170004)$ and $C=(137007,87762,274082,159609)$ the error is about $1.2 \%$.

1.3. Computations and a change of the hyperplane. Optimization problem (1.1) is convex and can be solved, for example, by interior point methods; see [24]. That is, for any $\epsilon>0$ the entries $\zeta_{j k}$ of the typical matrix $Z$ can be computed within relative error $\epsilon$ in time polynomial in $\ln (1 / \epsilon)$ and $m+n$.

Given $Z$, quantities $\operatorname{det} q \mid H, \mu$ and $\nu$ can be computed by linear algebra algorithms in $O\left(m^{2} n^{2}\right)$ time, since to compute the expectation of a polynomial with respect to the Gaussian measure one only needs to know the covariances of the variables; see Section 4.1. It may be convenient to replace the hyperplane $H \subset \mathbb{R}^{m+n}$ orthogonal to the null-space of $q$ by a coordinate hyperplane $L \subset \mathbb{R}^{m+n}$ defined by any of the equations $s_{j}=0$ or $t_{k}=0$. Indeed, if $L \subset \mathbb{R}^{m+n}$ is any hyperplane not containing the null-space of $q$, then the restriction $q \mid L$ is positive definite and one can consider the Gaussian probability measure in $L$ with the density proportional to $e^{-q}$. We prove in Lemma 3.1 below that the expectation of any polynomial in $s_{j}+t_{k}$ does not depend on the choice of $L$ and hence $\mu$ and $\nu$ can be defined as in Section 1.2 with $H$ replaced by $L$. We describe the dependence of $\operatorname{det} q \mid L$ on $L$ in Lemma 3.4. In particular, it follows that if $L$ is a coordinate hyperplane, then $\operatorname{det} q|H=(m+n) \operatorname{det} q| L$.

If we choose $L$ to be defined by the equation $t_{n}=0$, then we have an explicit formula for the matrix $Q$ of $q \mid L$ as follows:

$$
q(x)=\frac{1}{2}\langle x, Q x\rangle \quad \text { for } \quad x=\left(s_{1}, \ldots, s_{m} ; t_{1}, \ldots, t_{n-1}\right)
$$

where $\langle\cdot, \cdot\rangle$ is the standard scalar product and $Q=\left(q_{i l}\right)$ is the $(m+n-1) \times(m+n-1)$ symmetric matrix, where

$$
\begin{aligned}
q_{j(k+m)}=q_{(k+m) j} & =\zeta_{j k}^{2}+\zeta_{j k} \text { for } j=1, \ldots, m \text { and } k=1, \ldots, n-1, \\
q_{j j} & =r_{j}+\sum_{k=1}^{n} \zeta_{j k}^{2} \text { for } j=1, \ldots, m, \\
q_{(k+m)(k+m)} & =c_{k}+\sum_{j=1}^{n} \zeta_{j k}^{2} \quad \text { for } \quad k=1, \ldots, n-1,
\end{aligned}
$$

and all other entries $q_{i l}$ are zeros. Then $\operatorname{det} q \mid L=2^{1-m-n} \operatorname{det} Q$ and $Q^{-1}$ is the covariance matrix of $s_{1}, \ldots, s_{m} ; t_{1}, \ldots, t_{n-1}$.

\section{An integral REPRESEntation FOR THE NUMBER OF CONTINGENCY TABLES AND THE PLAN OF THE PROOF OF THEOREM 1.1}

In [5] we prove the following general result.

Theorem 2.1. Let $P \subset \mathbb{R}^{p}$ be a polyhedron defined by the system of linear equations $A x=b$, where $A$ is a $d \times p$ integer matrix with columns $a_{1}, \ldots, a_{p} \in \mathbb{Z}^{d}$ and $b \in \mathbb{Z}^{d}$ is an integer vector, and inequalities $x \geq 0$ (the inequalities are understood as being 
coordinate-wise). Suppose that $P$ is bounded and has a non-empty interior, that is, it contains a point $x=\left(\xi_{1}, \ldots, \xi_{p}\right)$ such that $\xi_{j}>0$ for $j=1, \ldots, p$. Then the function

$$
g(x)=\sum_{j=1}^{p}\left(\left(\xi_{j}+1\right) \ln \left(\xi_{j}+1\right)-\xi_{j} \ln \xi_{j}\right)
$$

attains its maximum on $P$ at a unique point $z=\left(\zeta_{1}, \ldots, \zeta_{p}\right)$ such that $\zeta_{j}>0$ for $j=1, \ldots, p$.

Let $\Pi \subset \mathbb{R}^{d}$ be the parallelepiped consisting of the points $t=\left(\tau_{1}, \ldots, \tau_{d}\right)$ such that

$$
-\pi \leq \tau_{k} \leq \pi \text { for } k=1, \ldots, d
$$

Then the number $\left|P \cap \mathbb{Z}^{p}\right|$ of integer points in $P$ can be written as

$$
\left|P \cap \mathbb{Z}^{p}\right|=\frac{e^{g(z)}}{(2 \pi)^{d}} \int_{\Pi} e^{-i\langle t, b\rangle} \prod_{j=1}^{p} \frac{1}{1+\zeta_{j}-\zeta_{j} e^{i\left\langle a_{j}, t\right\rangle}} d t
$$

where $\langle\cdot, \cdot\rangle$ is the standard scalar product in $\mathbb{R}^{d}$ and $i=\sqrt{-1}$.

The idea of the proof is as follows. Let $X=\left(x_{1}, \ldots, x_{p}\right)$ be a random vector of independent geometric random variables $x_{j}$ such that $\mathbf{E} x_{j}=\zeta_{j}$. Hence values of $X$ are non-negative integer vectors and we show in [5] that the probability mass function of $X$ is constant on the set $P \cap \mathbb{Z}^{p}$ and equals $e^{-g(z)}$ for every integer point in $P$. Letting $Y=A X$, we obtain

$$
\left|P \cap \mathbb{Z}^{p}\right|=e^{g(z)} \mathbf{P}\{X \in P\}=e^{g(z)} \mathbf{P}\{Y=b\}
$$

and the probability in question is written as the integral of the characteristic function of $Y$.

Since

$$
\sum_{j=1}^{p} \zeta_{j} a_{j}=b
$$

in a neighborhood of the origin $t=0$ the integrand can be written as

$$
\begin{aligned}
e^{-i\langle t, b\rangle} \prod_{j=1}^{p} & \frac{1}{1+\zeta_{j}-\zeta_{j} e^{i\left\langle a_{j}, t\right\rangle}} \\
=\exp \{ & -\frac{1}{2} \sum_{j=1}^{p}\left(\zeta_{j}^{2}+\zeta_{j}\right)\left\langle a_{j}, t\right\rangle^{2} \\
& -\frac{i}{6} \sum_{j=1}^{p} \zeta_{j}\left(\zeta_{j}+1\right)\left(2 \zeta_{j}+1\right)\left\langle a_{j}, t\right\rangle^{3} \\
& +\frac{1}{24} \sum_{j=1}^{p} \zeta_{j}\left(\zeta_{j}+1\right)\left(6 \zeta_{j}^{2}+6 \zeta_{j}+1\right)\left\langle a_{j}, t\right\rangle^{4} \\
& \left.+O\left(\sum_{j=1}^{p}\left(\zeta_{j}+1\right)^{5}\left\langle a_{j}, t\right\rangle^{5}\right)\right\}
\end{aligned}
$$

Note that the linear term is absent in the expansion. 
We obtain the following corollary.

Corollary 2.2. Let $R=\left(r_{1}, \ldots, r_{m}\right)$ and $C=\left(c_{1}, \ldots, c_{n}\right)$ be margins and let $Z=\left(\zeta_{j k}\right)$ be the typical matrix defined in Section 1.1. Let

$$
F(s, t)=\exp \left\{-i \sum_{j=1}^{m} r_{j} s_{j}-i \sum_{k=1}^{n} c_{k} t_{k}\right\} \prod_{\substack{1 \leq j \leq m \\ 1 \leq k \leq n}} \frac{1}{1+\zeta_{j k}-\zeta_{j k} e^{i\left(s_{j}+t_{k}\right)}} .
$$

Let $\Pi \subset \mathbb{R}^{m+n}$ be the parallelepiped consisting of the points $\left(s_{1}, \ldots, s_{m} ; t_{1}, \ldots, t_{n}\right)$ such that

$$
-\pi \leq s_{j}, t_{k} \leq \pi \text { for all } j, k .
$$

Let us identify $\mathbb{R}^{m+n-1}$ with the hyperplane $t_{n}=0$ in $\mathbb{R}^{m+n}$ and let $\Pi_{0} \subset \Pi$ be the facet of $\Pi$ defined by the equation $t_{n}=0$. Then

$$
\#(R, C)=\frac{e^{g(Z)}}{(2 \pi)^{m+n-1}} \int_{\Pi_{0}} F(s, t) d s d t,
$$

where dsdt is the Lebesgue measure in $\Pi_{0}$.

Proof. The number \# $(R, C)$ of non-negative integer $m \times n$ matrices with row sums $R$ and column sums $C$ is the number of integer points in the transportation polytope $P(R, C)$. We can define $P(R, C)$ by prescribing all row sums $r_{1}, \ldots, r_{m}$ and all but one column sums $c_{1}, \ldots, c_{n-1}$ of a non-negative $m \times n$ matrix. Applying Theorem 2.1, we get the desired integral representation.

From (2.1) we get the following expansion in the neighborhood of $s_{1}=\ldots=$ $s_{m}=t_{1}=\ldots=t_{n}=0$ :

$$
\begin{aligned}
F(s, t)=\exp \{ & -q(s, t)-i f(s, t)+h(s, t) \\
+ & \left.O\left(\sum_{j, k}\left(1+\zeta_{j k}\right)^{5}\left(s_{j}+t_{k}\right)^{5}\right)\right\},
\end{aligned}
$$

where $q, f$, and $h$ are defined by (1.5)-(1.6).

2.1. The plan of the proof of Theorem 1.1. First, we argue that it suffices to prove Theorem 1.1 under one additional assumption, namely, that the parameter $\tau$ in (1.3) is bounded by a polynomial in $m+n$ :

$$
\tau \leq(m+n)^{1 / \delta} \text { for some } \delta>0
$$

(for example, one can choose $\delta=1 / 10$ ). Indeed, it follows by results of 18 , (see Lemma 3 there) that for $\tau \geq(m n)^{2}$ the (properly normalized) volume vol $P(R, C)$ of the transportation polytope approximates the number of tables \# $(R, C)$ within a relative error of $O\left((m+n)^{-1}\right)$. Since $\operatorname{dim} P(R, C)=(m-1)(n-1)$ and

$$
\operatorname{vol} P(\alpha R, \alpha C)=\alpha^{(m-1)(n-1)} \operatorname{vol} P(R, C) \text { for } \quad \alpha>0 \text {, }
$$

to handle larger $\tau$ it suffices to show that the formula of Theorem 1.1 scales the right way if the margins $(R, C)$ get scaled $(R, C) \longmapsto(\alpha R, \alpha C)$ (and appropriately rounded, if the obtained margins are not integer). If $\tau$ is large enough, then scaling results in an approximate scaling $e^{g(Z)} \longmapsto \alpha^{m n} e^{g(Z)}, q \longmapsto \alpha^{2} q, f \longmapsto \alpha^{3} f$ and 
$h \longmapsto \alpha^{4} h$ and hence the value produced by the formula of Theorem 1.1 gets multiplied by roughly $\alpha^{(m-1)(n-1)}$, as desired. We provide necessary details in Section 8 .

To handle the case of $\tau$ bounded by a polynomial in $m+n$, we use the integral representation of Corollary 2.2 .

Let us define a neighborhood $\mathcal{U} \subset \Pi_{0}$ of the origin by

$$
\mathcal{U}=\left\{\left(s_{1}, \ldots, s_{m} ; t_{1}, \ldots, t_{n-1}\right): \quad\left|s_{j}\right|,\left|t_{k}\right| \leq \frac{\ln (m+n)}{\tau \sqrt{m+n}} \text { for all } j, k\right\} .
$$

We show that the integral of $F(s, t)$ over $\Pi_{0} \backslash \mathcal{U}$ is asymptotically negligible. Namely, in Section 7 we prove that the integral

$$
\int_{\Pi_{0} \backslash \mathcal{U}}|F(s, t)| d s d t
$$

is asymptotically negligible compared to the integral

$$
\int_{\mathcal{U}}|F(s, t)| d s d t
$$

In Section [6, we evaluate the integral

$$
\int_{\mathcal{U}} F(s, t) d s d t
$$

and show that it produces the asymptotic formula of Theorem 1.1. In particular, we show that (2.3) and (2.4) are of the same order, that is,

$$
\int_{\mathcal{U}}|F(s, t)| d s d t \leq \gamma(\delta)\left|\int_{\mathcal{U}} F(s, t) d s d t\right|
$$

for some constant $\gamma(\delta) \geq 1$. Hence the integral of $F(s, t)$ outside of $\mathcal{U}$ is indeed asymptotically irrelevant.

From (2.2), we deduce that

$$
F(s, t) \approx \exp \{-q(s, t)-i f(s, t)+h(s, t)\} \quad \text { for } \quad(s, t) \in \mathcal{U},
$$

where $q$ is defined by (1.5) and $f$ and $h$ are defined by (1.6), so that the contribution of the terms of order 5 and higher in (2.2) is asymptotically negligible in the integral (2.4). The integral of $e^{-q}$ over $\mathcal{U}$ produces the Gaussian term (1.7). However, both the cubic term $f(s, t)$ and the fourth-order term $h(s, t)$ contribute substantially to the integral, correcting the Gaussian term (1.7) by a constant factor.

Let us consider the Gaussian probability measure in the coordinate hyperplane $t_{n}=0$, which we identify with $\mathbb{R}^{m+n-1}$, with the density proportional to $e^{-q}$. In Section 5. we show that with respect to that measure, $h(s, t)$ remains, essentially, constant in the neighborhood $\mathcal{U}$ :

$$
h(s, t) \approx \mathbf{E} h=\nu \quad \text { almost everywhere in } \mathcal{U} .
$$

This allows us to conclude that asymptotically

$$
\int_{\mathcal{U}} \exp \{-q(s, t)-i f(s, t)+h(s, t)\} d s d t \approx e^{\nu} \int_{\mathcal{U}} \exp \{-q(s, t)+i f(s, t)\} d s d t .
$$


In Section 4, we show that $f(s, t)$ behaves, essentially, as a Gaussian random variable with respect to the probability measure in $\mathbb{R}^{m+n-1}$ with the density proportional to $e^{-q}$, so

$$
\begin{aligned}
\int_{\mathcal{U}} \exp \{-q(s, t)-i f(s, t)\} d s d t & \approx \int_{\mathbb{R}^{m+n-1}} \exp \{-q(s, t)-i f(s, t)\} d s d t \\
& \approx \exp \left\{-\frac{1}{2} \mathbf{E} f^{2}\right\} \int_{\mathbb{R}^{m+n-1}} e^{-q(s, t)} d s d t
\end{aligned}
$$

which concludes the computation of (2.4).

The results of Sections 4 and 5 are based on the analysis in Section 3 . In Section 3. we consider coordinate functions $s_{j}$ and $t_{k}$ as random variables with respect to the Gaussian probability measure on a hyperplane $L \subset \mathbb{R}^{m+n}$ not containing the null-space of $q$ with the density proportional to $e^{-q}$. We show that $s_{j_{1}}+t_{k_{1}}$ and $s_{j_{2}}+t_{k_{2}}$ are weakly correlated provided $j_{1} \neq j_{2}$ and $k_{1} \neq k_{2}$, that is,

$$
\begin{aligned}
\mathbf{E}\left|\left(s_{j_{1}}+t_{k_{1}}\right)\left(s_{j_{2}}+t_{k_{2}}\right)\right| & =O\left(\frac{1}{m n}\right) \quad \text { provided } \quad j_{1} \neq j_{2} \quad \text { and } \quad k_{1} \neq k_{2} \quad \text { and } \\
\mathbf{E}\left|\left(s_{j}+t_{k}\right)^{2}\right| & =O\left(\frac{1}{m+n}\right) \text { for all } j, k
\end{aligned}
$$

2.2. Notation. In what follows, we denote by $\gamma$, sometimes with an index or a list of parameters, a positive constant depending on the parameters. The actual value of $\gamma$ may change from line to line. The most common appearance will be $\gamma(\delta)$, a positive constant depending only on the $\delta$-smoothness constant $\delta$.

As usual, for two functions $f$ and $g$, where $g$ is non-negative, we say that $f=O(g)$ if $|f| \leq \gamma g$ for some constant $\gamma>0$ and that $f=\Omega(g)$ if $f \geq \gamma g$ for some constant $\gamma>0$

\section{Correlations}

Recall (see Section 1.2) that the quadratic form $q: \mathbb{R}^{m+n} \longrightarrow \mathbb{R}$ is defined by

$$
q(s, t)=\frac{1}{2} \sum_{\substack{1 \leq j \leq m \\ 1 \leq k \leq n}}\left(\zeta_{j k}^{2}+\zeta_{j k}\right)\left(s_{j}+t_{k}\right)^{2} \quad \text { for } \quad(s, t)=\left(s_{1}, \ldots, s_{m} ; t_{1}, \ldots t_{n}\right) .
$$

Let

$$
u=(\underbrace{1, \ldots, 1}_{m \text { times }} ; \underbrace{-1, \ldots,-1}_{n \text { times }}) .
$$

Let $L \subset \mathbb{R}^{m+n}$ be a hyperplane which does not contain $u$. Then the restriction $q \mid L$ of $q$ onto $L$ is a positive definite quadratic form and we can consider the Gaussian probability measure on $L$ with the density proportional to $e^{-q}$. We consider $s_{j}$ and $t_{k}$ as random variables on $L$ and estimate their covariances.

Lemma 3.1. For any $1 \leq j_{1}, j_{2} \leq m$ and any $1 \leq k_{1}, k_{2} \leq n$ the covariance

$$
\mathbf{E}\left(s_{j_{1}}+t_{k_{1}}\right)\left(s_{j_{2}}+t_{k_{2}}\right)
$$

is independent on the choice the hyperplane $L$, as long as $L$ does not contain $u$. 
Proof. Let $L_{1}, L_{2} \subset \mathbb{R}^{m+n}$ be two hyperplanes not containing $u$. Then we can define the projection $p r: L_{1} \longrightarrow L_{2}$ along the span of $u$, so that $\operatorname{pr}(x)$ for $x \in L_{1}$ is the unique $y \in L_{2}$ such that $y-x$ is a multiple of $u$. We note that $q(x)=q(x+t u)$ for all $x \in \mathbb{R}^{m+n}$ and all $t \in \mathbb{R}$. Therefore, the push-forward of the Gaussian probability measure on $L_{1}$ with the density proportional to $e^{-q}$ is the probability measure on $L_{2}$ with the density proportional to $e^{-q}$. Moreover, the value of $s_{j}+t_{k}$ does not change under the projection and hence the result follows.

The main result of this section is the following theorem.

Theorem 3.2. Let us fix a number $\delta>0$ and suppose that

$$
\tau \delta \leq \zeta_{j k} \leq \tau \text { for all } j, k
$$

and some $\tau>0$. Suppose that $\delta m \leq n$ and $\delta n \leq m$.

Let us define

$$
\begin{aligned}
a_{j} & =\sum_{k=1}^{n}\left(\zeta_{j k}^{2}+\zeta_{j k}\right) \quad \text { for } \quad j=1, \ldots, m \quad \text { and } \\
b_{k} & =\sum_{j=1}^{m}\left(\zeta_{j k}^{2}+\zeta_{j k}\right) \quad \text { for } \quad k=1, \ldots, n .
\end{aligned}
$$

Let

$$
\Delta=\frac{12}{\delta^{10}\left(\tau^{2}+\tau\right) m n} .
$$

Let $L \subset \mathbb{R}^{m+n}$ be a hyperplane not containing the null-space of $q$. Let us consider the Gaussian probability measure on $L$ with the density proportional to $e^{-q}$.

Then

$$
\begin{aligned}
& \left|\mathbf{E}\left(s_{j_{1}}+t_{k_{1}}\right)\left(s_{j_{2}}+t_{k_{2}}\right)\right| \leq \Delta \text { provided } j_{1} \neq j_{2} \text { and } k_{1} \neq k_{2}, \\
& \left|\mathbf{E}\left(s_{j}+t_{k_{1}}\right)\left(s_{j}+t_{k_{2}}\right)-\frac{1}{a_{j}}\right| \leq \Delta \text { provided } k_{1} \neq k_{2}, \\
& \left|\mathbf{E}\left(s_{j_{1}}+t_{k}\right)\left(s_{j_{2}}+t_{k}\right)-\frac{1}{b_{k}}\right| \leq \Delta \text { provided } j_{1} \neq j_{2} \text { and } \\
& \left|\mathbf{E}\left(s_{j}+t_{k}\right)^{2}-\frac{1}{a_{j}}-\frac{1}{b_{k}}\right| \leq \Delta \text { for all } j \text { and } k .
\end{aligned}
$$

The gist of Theorem 3.2 is that for a fixed $\delta>0$, while generally the covariance of $s_{j_{1}}+t_{k_{1}}$ and $s_{j_{2}}+t_{k_{2}}$ is $O\left(\tau^{-2}(m+n)^{-1}\right)$, it is only $O\left(\tau^{-2}(m+n)^{-2}\right)$ when $j_{1} \neq j_{2}$ and $k_{1} \neq k_{2}$.

In what follows, we will often deal with the following situation. Let $V$ be Euclidean space, let $\phi: V \longrightarrow \mathbb{R}$ be a positive semidefinite quadratic form and let $L \subset V$ be a subspace such that the restriction of $\phi$ onto $L$ is positive definite. We consider the Gaussian probability measure on $L$ with the density proportional to $e^{-\phi}$. For a polynomial (random variable) $f: L \longrightarrow \mathbb{R}$ we denote by $\mathbf{E}(f ; \phi \mid L)$ the expectation of $f$ with respect to that Gaussian measure. Instead of $\mathbf{E}(f ; \phi \mid V)$ we simply write $\mathbf{E}(f ; \phi)$. 
We will use the following standard facts. Suppose that there is a direct sum decomposition $V=L_{1}+L_{2}+\ldots+L_{k}$ where $L_{i}$ are pairwise orthogonal, such that

$$
\phi\left(x_{1}+\ldots+x_{k}\right)=\sum_{i=1}^{k} \phi\left(x_{i}\right) \quad \text { for all } \quad x_{i} \in L_{i} .
$$

In other words, the components $x_{i} \in L_{i}$ of a random point $x=x_{1}+\ldots+x_{k}, x \in V$, are independent. Then for any two linear functions $\ell_{1}, \ell_{2}: V \longrightarrow \mathbb{R}$ we have

$$
\mathbf{E}\left(\ell_{1} \ell_{2} ; \phi\right)=\sum_{i=1}^{k} \mathbf{E}\left(\ell_{1} \ell_{2} ; \phi \mid L_{i}\right) \text {. }
$$

Indeed, since

$$
\ell_{1,2}\left(x_{1}+\ldots+x_{k}\right)=\sum_{i=1}^{k} \ell_{1,2}\left(x_{i}\right)
$$

we obtain

$$
\mathbf{E}\left(\ell_{1} \ell_{2} ; \phi\right)=\sum_{i_{1}=1}^{k} \sum_{i_{2}=1}^{k} \mathbf{E}\left(\ell_{1}\left(x_{i_{1}}\right) \ell_{2}\left(x_{i_{2}}\right) ; \phi\right) .
$$

If $i_{1} \neq i_{2}$, we have

$$
\mathbf{E}\left(\ell_{1}\left(x_{i_{1}}\right) \ell_{2}\left(x_{i_{2}}\right) ; \phi\right)=\mathbf{E}\left(\ell_{1} ; \phi \mid L_{i_{1}}\right) \mathbf{E}\left(\ell_{2} ; \phi \mid L_{i_{2}}\right)=0
$$

while for $i_{1}=i_{2}=i$ we have

$$
\mathbf{E}\left(\ell_{1}\left(x_{i}\right) \ell_{2}\left(x_{i}\right) ; \phi\right)=\mathbf{E}\left(\ell_{1} \ell_{2} ; \phi \mid L_{i}\right) .
$$

We deduce Theorem 3.2 from the following statement.

Proposition 3.3. Let $m$ and $n$ be positive integers such that

$$
\delta m \leq n \text { and } \delta n \leq m \text { for some } 0<\delta<1 .
$$

For $j=1, \ldots, m$ and $k=1, \ldots, n$ let $\xi_{j k}$ be real numbers such that

$$
\alpha \leq \xi_{j k} \leq \beta \text { for all } j, k
$$

and some $\beta>\alpha>0$. Let

$$
\begin{aligned}
& a_{j}=\sum_{k=1}^{n} \xi_{j k} \quad \text { for } \quad j=1, \ldots, m \quad \text { and } \\
& b_{k}=\sum_{j=1}^{m} \xi_{j k} \quad \text { for } \quad k=1, \ldots, n .
\end{aligned}
$$

Let us define a quadratic form $\psi: \mathbb{R}^{m+n} \longrightarrow \mathbb{R}$ by

$$
\begin{aligned}
\psi(s, t)=\frac{1}{2} \sum_{\substack{1 \leq j \leq m \\
1 \leq k \leq n}} \xi_{j k}\left(\frac{s_{j}}{\sqrt{a_{j}}}+\frac{t_{k}}{\sqrt{b_{k}}}\right)^{2} & \\
& \quad \text { for } \quad(s, t)=\left(s_{1}, \ldots, s_{m} ; t_{1}, \ldots, t_{n}\right) .
\end{aligned}
$$

Let $L \subset \mathbb{R}^{m+n}$ be the hyperplane consisting of the points $\left(s_{1}, \ldots, s_{m} ; t_{1}, \ldots, t_{n}\right)$ such that

$$
\sum_{j=1}^{m} s_{j} \sqrt{a_{j}}=\sum_{k=1}^{n} t_{k} \sqrt{b_{k}} .
$$


Then the restriction $\psi \mid L$ of $\psi$ onto $L$ is positive definite and for

$$
\Delta=3\left(\frac{\beta}{\alpha}\right)^{7 / 2} \frac{1}{\sqrt{\delta m n}}
$$

we have

$\left|\mathbf{E}\left(s_{j}^{2} ; \psi \mid L\right)-1\right|,\left|\mathbf{E}\left(t_{k}^{2} ; \psi \mid L\right)-1\right| \leq \Delta$ for all $j, k$,

$\left|\mathbf{E}\left(s_{j_{1}} s_{j_{2}} ; \psi \mid L\right)\right|,\left|\mathbf{E}\left(t_{k_{1}} t_{k_{2}} ; \psi \mid L\right)\right| \leq \Delta$ for all $j_{1} \neq j_{2}$ and $k_{1} \neq k_{2}$,

$\left|\mathbf{E}\left(s_{j} t_{k} ; \psi \mid L\right)\right| \leq \Delta$ for all $j, k$.

Proof. Clearly, the null-space of $\psi$ is one dimensional and spanned by vector

$$
w=\left(\sqrt{a_{1}}, \ldots, \sqrt{a_{m}} ;-\sqrt{b_{1}}, \ldots,-\sqrt{b_{n}}\right) .
$$

We have $L=w^{\perp}$ and hence the restriction of $\psi$ onto $L$ is positive definite.

Next, we observe that

$$
v=\left(\sqrt{a_{1}}, \ldots, \sqrt{a_{m}} ; \sqrt{b_{1}}, \ldots, \sqrt{b_{n}}\right)
$$

is an eigenvector of $\psi$ with eigenvalue 1. Indeed, the gradient of $\psi(x)$ at $x=v$ is equal to $2 v$ :

$$
\begin{aligned}
& \left.\frac{\partial}{\partial s_{j}} \psi\right|_{s_{j}=\sqrt{a_{j}}, t_{k}=\sqrt{b_{k}}}=\frac{2}{\sqrt{a_{j}}} \sum_{k=1}^{n} \xi_{j k}=2 \sqrt{a_{j}} \text { and } \\
& \left.\frac{\partial}{\partial t_{k}} \psi\right|_{s_{j}=\sqrt{a_{j}}, t_{k}=\sqrt{b_{k}}}=\frac{2}{\sqrt{b_{k}}} \sum_{j=1}^{m} \xi_{j k}=2 \sqrt{b_{k}} .
\end{aligned}
$$

We write

$$
\psi(s, t)=\frac{1}{2} \sum_{j=1}^{m} s_{j}^{2}+\frac{1}{2} \sum_{k=1}^{n} t_{k}^{2}+\sum_{\substack{j=1, \ldots, m \\ k=1, \ldots, n}} \frac{\xi_{j k}}{\sqrt{a_{j}} \sqrt{b_{k}}} s_{j} t_{k} .
$$

Let

$$
c=a_{1}+\ldots+a_{m}=b_{1}+\ldots+b_{n}
$$

and let us consider another quadratic form $\phi: \mathbb{R}^{m+n} \longrightarrow \mathbb{R}$ defined by

$$
\phi(s, t)=\sum_{\substack{1 \leq j \leq m \\ 1 \leq k \leq n}} \frac{\sqrt{a_{j} b_{k}}}{c} s_{j} t_{k} \quad \text { for } \quad(s, t)=\left(s_{1}, \ldots, s_{m} ; t_{1}, \ldots, t_{n}\right) .
$$

Clearly, $\phi(s, t)$ is a form of rank 2 . Its non-zero eigenvalues are $-1 / 2$ with the eigenspace spanned by $w$ and $1 / 2$ with the eigenspace spanned by $v$.

Let us define a subspace $L_{0} \subset \mathbb{R}^{m+n}$ of codimension 2 by

$$
L_{0}=(v, w)^{\perp} .
$$

In other words, $L_{0}$ consists of the points $\left(s_{1}, \ldots, s_{m} ; t_{1}, \ldots, t_{n}\right)$ such that

$$
\sum_{j=1}^{m} s_{j} \sqrt{a_{j}}=\sum_{k=1}^{n} t_{k} \sqrt{b_{k}}=0 .
$$

In particular,

$$
\phi(s, t)=0 \quad \text { for all } \quad(s, t) \in L_{0} .
$$


Let us define a quadratic form

$$
\tilde{\psi}=\psi-\epsilon^{2} \phi \quad \text { for } \quad \epsilon=\frac{\alpha}{\beta} .
$$

We note that $\tilde{\psi}$ is positive definite. Indeed, $w$ and $v$ are eigenvectors of $\tilde{\psi}$ with the eigenvalues $\epsilon^{2} / 2>0$ and $1-\epsilon^{2} / 2>0$, respectively, and $\tilde{\psi}$ coincides with $\psi$ on the subspace $L_{0}=(v, w)^{\perp}$, where $\psi$ is positive definite. Our immediate goal is to bound the covariances

$$
\mathbf{E}\left(s_{j_{1}} s_{j_{2}} ; \tilde{\psi}\right), \mathbf{E}\left(t_{k_{1}} t_{k_{2}} ; \tilde{\psi}\right) \text { and } \mathbf{E}\left(s_{j} t_{k} ; \tilde{\psi}\right) .
$$

We can write

$$
\tilde{\psi}(x)=\frac{1}{2}\langle x,(I+P) x\rangle \quad \text { for } \quad x=(s, t),
$$

where $I$ is the $(m+n) \times(m+n)$ identity matrix, $P=\left(p_{i l}\right)$ is a symmetric $(m+n) \times(m+n)$ matrix with zero diagonal and $\langle\cdot, \cdot\rangle$ is the standard scalar product in $\mathbb{R}^{m+n}$. Since

$$
\begin{gathered}
\alpha n \leq a_{j} \leq \beta n \text { for } j=1, \ldots, m, \\
\alpha m \leq b_{k} \leq \beta m \text { for } k=1, \ldots, n, \quad \text { and } \\
c \geq \alpha m n,
\end{gathered}
$$

by (3.1) - (3.3), for the entries $p_{i l}$ of $P$ we have

$$
0 \leq p_{i l} \leq \frac{\beta}{\alpha \sqrt{m n}}=\frac{1}{\epsilon \sqrt{m n}} \text { for all } i, l .
$$

Furthermore, $v$ is the Perron-Frobenius eigenvector of $P$ with the corresponding eigenvalue $1-\epsilon^{2}$.

Let us bound the entries of a positive integer power $P^{d}=\left(p_{i l}^{(d)}\right)$ of $P$. Let

$$
\kappa=\frac{\beta}{\alpha^{3 / 2} \delta^{1 / 4}(m n)^{3 / 4}}, \quad \text { and let } \quad y=\kappa v, \quad y=\left(\eta_{1}, \ldots, \eta_{m+n}\right) .
$$

From (3.4) we conclude that

$$
a_{j}, b_{k} \geq \alpha \sqrt{\delta m n} \text { for all } j, k
$$

and hence by (3.5)

$$
p_{i l} \leq \eta_{i} \text { for all } i, l .
$$

Similarly, from (3.4), we conclude

$$
a_{j}, b_{k} \leq \beta \sqrt{m n / \delta} \text { for all } j, k
$$

and hence

$$
\eta_{i} \leq \frac{\beta^{3 / 2}}{\alpha^{3 / 2} \sqrt{\delta m n}}=\frac{1}{\epsilon^{3 / 2} \sqrt{\delta m n}} \text { for all } i
$$


Besides, $y$ is an eigenvector of $P^{d}$ with the eigenvalue $\left(1-\epsilon^{2}\right)^{d}$. Therefore, for $d \geq 0$ we have

$$
\begin{aligned}
p_{i l}^{(d+1)} & =\sum_{j=1}^{m+n} p_{i j}^{(d)} p_{j l} \\
& \leq \sum_{j=1}^{m+n} p_{i j}^{(d)} \eta_{j}=\left(1-\epsilon^{2}\right)^{d} \eta_{i} \\
& \leq\left(1-\epsilon^{2}\right)^{d} \frac{1}{\epsilon^{3 / 2} \sqrt{\delta m n}} .
\end{aligned}
$$

Consequently, the series

$$
(I+P)^{-1}=I+\sum_{d=1}^{+\infty}(-1)^{d} P^{d}
$$

converges absolutely and we can bound the entries of $Q=(I+P)^{-1}, q=\left(q_{i l}\right)$, by

$$
\begin{aligned}
& \left|q_{i l}\right| \leq \frac{1}{\epsilon^{2}} \frac{1}{\epsilon^{3 / 2} \sqrt{\delta m n}}=\frac{1}{\epsilon^{7 / 2} \sqrt{\delta m n}} \text { if } i \neq l \\
& \text { and } \\
& \left|q_{i i}-1\right| \leq \frac{1}{\epsilon^{7 / 2} \sqrt{\delta m n}}
\end{aligned}
$$

On the other hand, $Q$ is the matrix of covariances of functions $s_{1}, \ldots, s_{m} ; t_{1}, \ldots, t_{n}$, so we have

$$
\begin{aligned}
& \left|\mathbf{E}\left(s_{j}^{2} ; \tilde{\psi}\right)-1\right|,\left|\mathbf{E}\left(t_{k}^{2} ; \tilde{\psi}\right)-1\right| \leq \frac{1}{\epsilon^{7 / 2} \sqrt{\delta m n}} \text { for all } j, k, \\
& \left|\mathbf{E}\left(s_{j_{1}} s_{j_{2}} ; \tilde{\psi}\right)\right| \leq \frac{1}{\epsilon^{7 / 2} \sqrt{\delta m n}} \text { if } j_{1} \neq j_{2}, \\
& \left|\mathbf{E}\left(t_{k_{1}} t_{k_{2}} ; \tilde{\psi}\right)\right| \leq \frac{1}{\epsilon^{7 / 2} \sqrt{\delta m n}} \text { if } k_{1} \neq k_{2} \\
& \quad \text { and } \\
& \left|\mathbf{E}\left(s_{j} t_{k} ; \tilde{\psi}\right)\right| \leq \frac{1}{\epsilon^{7 / 2} \sqrt{\delta m n}} \text { for all } j, k .
\end{aligned}
$$

Now we go from $\tilde{\psi}$ back to $\psi$. Since $v$ and $w$ are eigenvectors of $\tilde{\psi}$ and since $L_{0}=(v, w)^{\perp}$, for any linear functions $\ell_{1}, \ell_{2}: \mathbb{R}^{m+n} \longrightarrow \mathbb{R}$ we have

$$
\mathbf{E}\left(\ell_{1} \ell_{2} ; \tilde{\psi} \mid L_{0}\right)=\mathbf{E}\left(\ell_{1} \ell_{2} ; \tilde{\psi}\right)-\mathbf{E}\left(\ell_{1} \ell_{2} ; \tilde{\psi} \mid \operatorname{span}(w)\right)-\mathbf{E}\left(\ell_{1} \ell_{2} ; \tilde{\psi} \mid \operatorname{span}(v)\right) \text {. }
$$

On the other hand, since $\psi$ and $\tilde{\psi}$ coincide on $L_{0}$, we have

$$
\mathbf{E}\left(\ell_{1} \ell_{2} ; \psi \mid L_{0}\right)=\mathbf{E}\left(\ell_{1} \ell_{2} ; \tilde{\psi} \mid L_{0}\right) .
$$

Finally, since $v$ is an eigenvector of $\psi$ and $L_{0}$ is the orthogonal complement to $v$ in $L$, we have

$$
\mathbf{E}\left(\ell_{1} \ell_{2} ; \psi \mid L\right)=\mathbf{E}\left(\ell_{1} \ell_{2} ; \psi \mid L_{0}\right)+\mathbf{E}\left(\ell_{1} \ell_{2} ; \psi \mid \operatorname{span}(v)\right) .
$$


Therefore,

$$
\begin{aligned}
\mathbf{E}\left(\ell_{1} \ell_{2} ; \psi \mid L\right)= & \mathbf{E}\left(\ell_{1} \ell_{2} ; \tilde{\psi}\right)-\mathbf{E}\left(\ell_{1} \ell_{2} ; \tilde{\psi} \mid \operatorname{span}(w)\right) \\
& -\mathbf{E}\left(\ell_{1} \ell_{2} ; \tilde{\psi} \mid \operatorname{span}(v)\right)+\mathbf{E}\left(\ell_{1} \ell_{2} ; \psi \mid \operatorname{span}(v)\right)
\end{aligned}
$$

We note that the gradient of function $s_{j}$ restricted onto $\operatorname{span}(w)$ is $\sqrt{a_{j} / 2 c}$. Since $w$ is an eigenvector of $\tilde{\psi}$ with eigenvalue $\epsilon^{2} / 2$, we have

$$
\mathbf{E}\left(s_{j_{1}} s_{j_{2}} ; \tilde{\psi} \mid \operatorname{span}(w)\right)=\frac{\sqrt{a_{j_{1} a_{j_{2}}}}}{2 \epsilon^{2} c} \leq \frac{1}{2 \epsilon^{3} \sqrt{\delta m n}} \text { for all } j_{1}, j_{2} .
$$

Similarly,

$$
\mathbf{E}\left(t_{k_{1}} t_{k_{2}} ; \tilde{\psi} \mid \operatorname{span}(w)\right)=\frac{\sqrt{b_{k_{1}} b_{k_{2}}}}{2 \epsilon^{2} c} \leq \frac{1}{2 \epsilon^{3} \sqrt{\delta m n}} \text { for all } k_{1}, k_{2}
$$

and

$$
\mathbf{E}\left(s_{j} t_{k} ; \tilde{\psi} \mid \operatorname{span}(w)\right)=-\frac{\sqrt{a_{j} b_{k}}}{2 \epsilon^{2} c} \geq-\frac{1}{2 \epsilon^{3} \sqrt{m n}} \text { for all } j, k \text {. }
$$

Since $v$ is an eigenvector of $\tilde{\psi}$ with eigenvalue $1-\epsilon^{2} / 2 \geq 1 / 2$, we obtain

$$
\mathbf{E}\left(s_{j_{1}} s_{j_{2}} ; \tilde{\psi} \mid \operatorname{span}(v)\right)=\frac{\sqrt{a_{j_{1}} a_{j_{2}}}}{4\left(1-\epsilon^{2} / 2\right) c} \leq \frac{1}{2 \epsilon \sqrt{\delta m n}} \text { for all } j_{1}, j_{2} .
$$

Similarly,

$$
\mathbf{E}\left(t_{k_{1}} t_{k_{2}} ; \tilde{\psi} \mid \operatorname{span}(v)\right)=\frac{\sqrt{b_{k_{1}} b_{k_{2}}}}{4\left(1-\epsilon^{2} / 2\right) c} \leq \frac{1}{2 \epsilon \sqrt{\delta m n}} \text { for all } k_{1}, k_{2}
$$

and

$$
\mathbf{E}\left(s_{j} t_{k} ; \tilde{\psi} \mid \operatorname{span}(v)\right)=\frac{\sqrt{a_{j} b_{k}}}{4\left(1-\epsilon^{2} / 2\right) c} \leq \frac{1}{2 \epsilon \sqrt{m n}} \text { for all } j, k \text {. }
$$

Since $v$ is an eigenvector of $\psi$ with eigenvalue 1 , we get

$$
\mathbf{E}\left(s_{j_{1}} s_{j_{2}} ; \psi \mid \operatorname{span}(v)\right)=\frac{\sqrt{a_{j_{1}} a_{j_{2}}}}{4 c} \leq \frac{1}{4 \epsilon \sqrt{\delta m n}} \text { for all } j_{1}, j_{2} .
$$

Similarly,

$$
\mathbf{E}\left(t_{k_{1}} t_{k_{2}} ; \psi \mid \operatorname{span}(v)\right)=\frac{\sqrt{b_{k_{1}} b_{k_{2}}}}{4 c} \leq \frac{1}{4 \epsilon \sqrt{\delta m n}} \text { for all } k_{1}, k_{2}
$$

and

$$
\mathbf{E}\left(s_{j} t_{k} ; \psi \mid \operatorname{span}(v)\right)=\frac{\sqrt{a_{j} b_{k}}}{4 c} \leq \frac{1}{4 \epsilon \sqrt{m n}} \text { for all } j, k .
$$

Combining (3.6) and (3.7), we complete the proof. 
Now we are ready to prove Theorem 3.2 .

3.1. Proof of Theorem 3.2. Let us define

$$
\xi_{j k}=\zeta_{j k}^{2}+\zeta_{j k} \text { for all } j, k .
$$

Hence we have

$$
\begin{aligned}
& \alpha \leq \xi_{j k} \leq \beta \quad \text { for all } j, k, \quad \text { where } \\
& \alpha=\tau \delta+\tau^{2} \delta^{2} \quad \text { and } \beta=\tau+\tau^{2} .
\end{aligned}
$$

We have

$$
\frac{\beta}{\alpha}=\frac{\tau+\tau^{2}}{\tau \delta+\tau^{2} \delta^{2}}=\frac{1+\tau}{\delta+\tau \delta^{2}} \leq \frac{1}{\delta^{2}} .
$$

Let

$$
a_{j}=\sum_{k=1}^{n} \xi_{j k} \quad \text { and } \quad b_{k}=\sum_{j=1}^{m} \xi_{j k}
$$

In particular, we have

$$
\begin{aligned}
& a_{j} \geq\left(\tau+\tau^{2}\right) \delta^{2} n \quad \text { for } \quad j=1, \ldots, m \quad \text { and } \\
& b_{k} \geq\left(\tau+\tau^{2}\right) \delta^{2} m \text { for } \quad k=1, \ldots, n .
\end{aligned}
$$

We apply Proposition 3.3 to the quadratic form

$$
\psi=\frac{1}{2} \sum_{\substack{1 \leq j \leq m \\ 1 \leq k \leq n}} \xi_{j k}\left(\frac{s_{j}}{\sqrt{a_{j}}}+\frac{t_{k}}{\sqrt{b_{k}}}\right)^{2}
$$

and the hyperplane $L_{1} \subset \mathbb{R}^{m+n}$ defined by the equation

$$
\sum_{j=1}^{m} s_{j} \sqrt{a_{j}}=\sum_{k=1}^{n} t_{k} \sqrt{b_{k}} .
$$

Let us consider a linear transformation

$$
\left(s_{1}, \ldots, s_{m} ; t_{1}, \ldots, t_{n}\right) \longmapsto\left(s_{1} \sqrt{a_{1}}, \ldots, s_{m} \sqrt{a_{m}} ; t_{1} \sqrt{b_{1}}, \ldots, t_{n} \sqrt{b_{n}}\right)
$$

and the hyperplane $L_{2} \subset \mathbb{R}^{m+n}$ defined by the equation

$$
\sum_{j=1}^{m} a_{j} s_{j}=\sum_{k=1}^{n} b_{k} t_{k}
$$

Then $L_{2}$ is mapped onto $L_{1}$ and the push-forward of the Gaussian probability measure on $L_{2}$ with the density proportional to $e^{-q}$ is the Gaussian probability measure on $L_{1}$ with the density proportional to $e^{-\psi}$.

We have

$$
\begin{aligned}
& \mathbf{E}\left(s_{j_{1}} s_{j_{2}} ; q \mid L_{2}\right)=\frac{1}{\sqrt{a_{j_{1}} a_{j_{2}}}} \mathbf{E}\left(s_{j_{1}} s_{j_{2}} ; \psi \mid L_{1}\right) \quad \text { for all } j_{1}, j_{2}, \\
& \mathbf{E}\left(t_{k_{1}} t_{k_{2}} ; q \mid L_{2}\right)=\frac{1}{\sqrt{b_{k_{1}} b_{k_{2}}}} \mathbf{E}\left(t_{k_{1}} t_{k_{2}} ; \psi \mid L_{1}\right) \quad \text { for all } k_{1}, k_{2}, \quad \text { and } \\
& \mathbf{E}\left(s_{j} t_{k} ; q \mid L_{2}\right)=\frac{1}{\sqrt{a_{j} b_{k}}} \mathbf{E}\left(s_{j} t_{k} ; \psi \mid L_{1}\right) \quad \text { for all } j, k .
\end{aligned}
$$


By (3.8), we have $\beta / \alpha \leq \delta^{-2}$. Since by Lemma 3.1, for any hyperplane $L \subset \mathbb{R}^{m+n}$ not containing $u$ we have

$$
\mathbf{E}\left(\left(s_{j_{1}}+t_{k_{1}}\right)\left(s_{j_{2}}+t_{k_{2}}\right) ; q \mid L\right)=\mathbf{E}\left(\left(s_{j_{1}}+t_{k_{1}}\right)\left(s_{j_{2}}+t_{k_{2}}\right) ; q \mid L_{2}\right),
$$

the proof follows by Proposition 3.3 applied to $L_{1}$ and $\psi$ and (3.8)- 3.10 ).

We will need the following result.

Lemma 3.4. Let $V$ be Euclidean space and let $q: V \longrightarrow \mathbb{R}$ be a quadratic form such that $\operatorname{rank} q=\operatorname{dim} V-1$. Let $v \in V$ be the unit eigenvector of $q$ with the eigenvalue 0 and let $H=v^{\perp}$ be the orthogonal complement of $v$. Then for a unit vector $u \in V$ we have

$$
\operatorname{det} q\left|u^{\perp}=\langle u, v\rangle^{2} \operatorname{det} q\right| H .
$$

Proof. This is Lemma 2.3 of 2 .

We apply Lemma 3.4 in the following situation. Let $V=\mathbb{R}^{m+n}$ and let $q$ be defined by (1.5). Let $L$ be a coordinate hyperplane defined by one of the equations $s_{j}=0$ or $t_{k}=0$. Then

$$
\operatorname{det} q\left|L=\frac{1}{m+n} \operatorname{det} q\right| H
$$

In particular, the value of $\operatorname{det} q \mid L$ does not depend on the choice of the coordinate hyperplane.

Finally, we need the following result.

Lemma 3.5. Let $q_{0}: \mathbb{R}^{m+n} \longrightarrow \mathbb{R}$ be the quadratic form defined by the formula

$$
q_{0}(s, t)=\frac{1}{2} \sum_{\substack{1 \leq j \leq m \\ 1 \leq k \leq n}}\left(s_{j}+t_{k}\right)^{2} .
$$

Then the eigenspaces of $q_{0}$ are as follows:

The one-dimensional eigenspace $E_{1}$ with the eigenvalue 0 spanned by vector

$$
u=(\underbrace{1, \ldots, 1}_{m \text { times }} ; \underbrace{-1, \ldots,-1}_{n \text { times }})
$$

the $(n-1)$-dimensional eigenspace $E_{2}$ with the eigenvalue $m / 2$ consisting of the vectors such that

$$
\sum_{k=1}^{n} t_{k}=0 \quad \text { and } \quad s_{1}=\ldots=s_{m}=0
$$

the $(m-1)$-dimensional eigenspace $E_{3}$ with the eigenvalue $n / 2$ consisting of the vectors such that

$$
\sum_{j=1}^{m} s_{j}=0 \quad \text { and } \quad t_{1}=\ldots=t_{n}=0
$$

and

the one-dimensional eigenspace $E_{4}$ with the eigenvalue $(m+n) / 2$ spanned by vector

$$
v=(\underbrace{n, \ldots, n}_{m \text { times }} ; \underbrace{m, \ldots, m}_{n \text { times }}) .
$$


Proof. Clearly, $E_{1}$ is the eigenspace with the eigenvalue 0 . It is then straightforward to check that the gradient of $q_{0}$ at $x=(s, t)$ equals $m x$ for $x \in E_{2}$, equals $n x$ for $x \in E_{3}$ and equals $(m+n) x$ for $x \in E_{4}$.

\section{The THIRD DEGREe TERM}

In this section we prove the following main result.

Theorem 4.1. Let $u_{j k}, j=1, \ldots, m$ and $k=1, \ldots, n$ be Gaussian random variables such that

$$
\mathbf{E} u_{j k}=0 \quad \text { for all } \quad j, k \text {. }
$$

Suppose further that for some $\theta>0$

$$
\begin{aligned}
& \mathbf{E} u_{j k}^{2} \leq \frac{\theta}{m+n} \text { for all } j, k \text { and } \\
& \left|\mathbf{E} u_{j_{1} k_{1}} u_{j_{2} k_{2}}\right| \leq \frac{\theta}{(m+n)^{2}} \quad \text { provided } j_{1} \neq j_{2} \text { and } k_{1} \neq k_{2} .
\end{aligned}
$$

Let

$$
U=\sum_{\substack{1 \leq j \leq m \\ 1 \leq k \leq n}} u_{j k}^{3} .
$$

Then for some constant $\gamma(\theta)>0$ and any $0<\epsilon \leq 1 / 2$ we have

$$
\left|\mathbf{E} \exp \{i U\}-\exp \left\{-\frac{1}{2} \mathbf{E} U^{2}\right\}\right| \leq \epsilon
$$

provided

$$
m+n \geq\left(\frac{1}{\epsilon}\right)^{\gamma(\theta)}
$$

Besides,

$$
\mathbf{E} U^{2} \leq \gamma(\theta)
$$

for some constant $\gamma(\theta)>0$. Here $i=\sqrt{-1}$.

We will apply Theorem 4.1 in the following situation. Let $q: \mathbb{R}^{m+n} \longrightarrow \mathbb{R}$ be the quadratic form defined by (1.5). Let $L \subset \mathbb{R}^{m+n}$ be a hyperplane not containing the null-space of $q$. Let us fix the Gaussian probability measure on $L$ with the density proportional to $e^{-q}$. We define random variables

$$
u_{j k}=\sqrt[3]{\frac{\zeta_{j k}\left(\zeta_{j k}+1\right)\left(2 \zeta_{j k}+1\right)}{6}}\left(s_{j}+t_{k}\right)
$$

where $s_{1}, \ldots, s_{m} ; t_{1}, \ldots, t_{n}$ are the coordinates of a point in $L$. Then we have

$$
U=\sum_{\substack{1 \leq j \leq m \\ 1 \leq k \leq n}} u_{j k}^{3}=f(s, t)
$$

for $f$ defined by (1.6). 
4.1. The expectation of a product of Gaussian random variables. We will use the famous Wick's formula; see, for example, [26. Let $w_{1}, \ldots, w_{l}$ be Gaussian random variables such that

$$
\mathbf{E} w_{1}=\ldots=\mathbf{E} w_{l}=0 .
$$

Then

$$
\begin{aligned}
& \mathbf{E} w_{1} \cdots w_{l}=0 \quad \text { if } \quad l=2 r+1 \quad \text { is odd } \quad \text { and } \\
& \mathbf{E} w_{1} \cdots w_{l}=\sum\left(\mathbf{E} w_{i_{1}} w_{i_{2}}\right) \cdots\left(\mathbf{E} w_{i_{2 r-1}} w_{i_{2 r}}\right) \quad \text { if } \quad l=2 r \quad \text { is even }
\end{aligned}
$$

and the sum is taken over all $(2 r) ! / r ! 2^{r}$ unordered partitions of the set of indices $\{1, \ldots, 2 r\}$ into $r$ pairwise disjoint unordered pairs $\left\{i_{1}, i_{2}\right\}, \ldots,\left\{i_{2 r-1}, i_{2 r}\right\}$. Such a partition is called a matching of the random variables $w_{1}, \ldots, w_{l}$. We say that $w_{i}$ and $w_{j}$ are matched if they form a pair in the matching.

In particular,

$$
\mathbf{E} w^{2 r}=\frac{(2 r) !}{r ! 2^{r}}\left(\mathbf{E} w^{2}\right)^{r}
$$

We will also use that

$$
\mathbf{E}\left(w_{1}^{3} w_{2}^{3}\right)=9\left(\mathbf{E} w_{1}^{2}\right)\left(\mathbf{E} w_{2}^{2}\right)\left(\mathbf{E} w_{1} w_{2}\right)+6\left(\mathbf{E} w_{1} w_{2}\right)^{3}
$$

and later in Section 5 that

$$
\begin{aligned}
\operatorname{cov}\left(w_{1}^{4}, w_{2}^{4}\right)= & \mathbf{E}\left(w_{1}^{4} w_{2}^{4}\right)-\left(\mathbf{E} w_{1}^{4}\right)\left(\mathbf{E} w_{2}^{4}\right) \\
= & 9\left(\mathbf{E} w_{1}^{2}\right)^{2}\left(\mathbf{E} w_{2}^{2}\right)^{2}+72\left(\mathbf{E} w_{1} w_{2}\right)^{2}\left(\mathbf{E} w_{1}^{2}\right)\left(\mathbf{E} w_{2}^{2}\right) \\
& \quad+24\left(\mathbf{E} w_{1} w_{2}\right)^{4}-9\left(\mathbf{E} w_{1}^{2}\right)^{2}\left(\mathbf{E} w_{2}^{2}\right)^{2} \\
= & 72\left(\mathbf{E} w_{1} w_{2}\right)^{2}\left(\mathbf{E} w_{1}^{2}\right)\left(\mathbf{E} w_{2}^{2}\right)+24\left(\mathbf{E} w_{1} w_{2}\right)^{4} .
\end{aligned}
$$

All implied constants in the " $O$ " notation in this section are absolute.

4.2. Auxiliary random variables $v_{j k}$. For the Gaussian random variables $\left\{u_{j k}\right\}$ of Theorem 4.1, let us define Gaussian random variables $\left\{v_{j k}\right\}$, where $j=1, \ldots, m$ and $k=1, \ldots, n$ such that

$$
\begin{aligned}
& \mathbf{E} v_{j k}=0 \text { for all } j, k \text { and } \\
& \mathbf{E}\left(v_{j_{1} k_{1}} v_{j_{2} k_{2}}\right)=\mathbf{E}\left(u_{j_{1} k_{1}}^{3} u_{j_{2} k_{2}}^{3}\right) \quad \text { for all } j_{1}, j_{2} \text { and } k_{1}, k_{2} .
\end{aligned}
$$

We say that the random variables $u_{j_{1} k_{1}}$ and $u_{j_{2} k_{2}}$ in Theorem 4.1 are weakly correlated if $j_{1} \neq j_{2}$ and $k_{1} \neq k_{2}$ and that they are strongly correlated otherwise. Similarly, we say that $v_{j_{1} k_{1}}$ and $v_{j_{2} k_{2}}$ are weakly correlated if $j_{1} \neq j_{2}$ and $k_{1} \neq k_{2}$ and are strongly correlated otherwise.

By (4.2),

$$
\begin{aligned}
& \mathbf{E} u_{j_{1} k_{1}}^{3} u_{j_{2} k_{2}}^{3}=\mathbf{E} v_{j_{1} k_{1}} v_{j_{2} k_{2}} \\
& \quad= \begin{cases}O\left(\theta^{3} /(m+n)^{4}\right) & \text { if } u_{j_{1} k_{1}}, u_{j_{2} k_{2}} \text { are weakly correlated } \\
O\left(\theta^{3} /(m+n)^{3}\right) & \text { if } u_{j_{1} k_{1}}, u_{j_{2} k_{2}} \text { are strongly correlated }\end{cases}
\end{aligned}
$$

Since the number of weakly correlated pairs is $O\left(m^{2} n^{2}\right)$ while the number of strongly correlated pairs is $O\left(m^{2} n+n^{2} m\right)$, we obtain that

$$
\mathbf{E} V^{2}=\mathbf{E} U^{2}=O\left(\theta^{3}\right) \text {. }
$$


4.3. Representation of monomials by graphs. Let $x_{j k}, j=1, \ldots, m, k=$ $1, \ldots, n$, be formal commuting variables. We interpret a monomial in $x_{j k}$ combinatorially, as a weighted graph. Let $K_{m, n}$ be the complete bipartite graph with $m+n$ vertices and $m n$ edges $(j, k)$ for $j=1, \ldots, m$ and $k=1, \ldots, n$. A weighted graph $G$ is a set of edges $(j, k)$ of $K_{m, n}$ with positive integer weights $\alpha_{j k}$ on it. With $G$, we associate a monomial

$$
t_{G}(x)=\prod_{(j, k) \in G} x_{j k}^{\alpha_{j k}} .
$$

The weight $\sum_{e \in G} \alpha_{e}$ of $G$ is the degree of the monomial. We observe that for any $p$ there are not more than $r^{O(r)}(m+n)^{p}$ distinct weighted graphs of weight $2 r$ and $p$ vertices. We note that pairs of variables $u_{j_{1} k_{1}}$ and $u_{j_{2} k_{2}}$ and pairs of variables $v_{j_{1} k_{1}}$ and $v_{j_{2} k_{2}}$ corresponding to the edges $\left(j_{1}, k_{1}\right)$ and $\left(j_{2}, k_{2}\right)$ in different connected components are weakly correlated.

Lemma 4.2. Given a graph $G$ of weight $2 r, r>1$, let us represent it as a vertexdisjoint union

$$
G=G_{0} \cup G_{1},
$$

where $G_{0}$ consists of $s$ isolated edges of weight 1 each and $G_{1}$ is a graph with no isolated edges of weight 1 (we may have $s=0$ and no $G_{0}$ ).

Then

(1) We have

$$
\begin{aligned}
\left|\mathbf{E} t_{G}\left(u_{j k}^{3}\right)\right| & \leq \frac{r^{O(r)} \theta^{3 r}}{(m+n)^{3 r+s / 2}} \quad \text { and } \\
\left|\mathbf{E} t_{G}\left(v_{j k}\right)\right| & \leq \frac{r^{O(r)} \theta^{3 r}}{(m+n)^{3 r+s / 2}} .
\end{aligned}
$$

Additionally, if $s$ is odd, then

$$
\left|\mathbf{E} t_{G}\left(u_{j k}^{3}\right)\right|,\left|\mathbf{E} t_{G}\left(v_{j k}\right)\right| \leq \frac{r^{O(r)} \theta^{3 r}}{(m+n)^{3 r+(s+1) / 2}} .
$$

(2) If $s$ is even and $G_{1}$ has $r-s / 2$ connected components, each consisting of a pair of edges of weight 1 each sharing one common vertex, then the number of vertices of $G$ is

$$
3 r+\frac{s}{2}
$$

In all other cases, $G$ has strictly fewer than $3 r+s / 2$ vertices.

(3) Suppose that $s$ is even and that $G_{1}$ has $r-s / 2$ connected components, each consisting of a pair of edges of weight 1 each sharing one common vertex. Then

$$
\left|\mathbf{E} t_{G}\left(u_{j k}^{3}\right)-\mathbf{E} t_{G}\left(v_{j k}\right)\right| \leq \frac{r^{O(r)} \theta^{3 r}}{(m+n)^{3 r+s / 2+1}} .
$$

Proof. To prove part (1), we use Wick's formula of Section 4.1. Since for each isolated edge $\left(j_{1}, k_{1}\right) \in G_{0}$, at least one of the three copies of the random variable $u_{j_{1} k_{1}}$ has to be matched with a copy of the variable $u_{j_{2} k_{2}}$ indexed by an edge $\left(j_{2}, k_{2}\right)$ in a different connected component, we conclude that each matching of the multiset

$$
\left\{u_{j k}, u_{j k}, u_{j k}:(j, k) \in G\right\}
$$


contains at least $s / 2$ weakly correlated pairs of variables and hence

$$
\left|\mathbf{E} t_{G}\left(u_{j k}^{3}\right)\right| \leq r^{O(r)}\left(\frac{\theta}{(m+n)^{2}}\right)^{s / 2}\left(\frac{\theta}{m+n}\right)^{3 r-s / 2} .
$$

Moreover, if $s$ is odd, the number of weakly correlated pairs in every matching is at least $(s+1) / 2$ and hence

$$
\left|\mathbf{E} t_{G}\left(u_{j k}^{3}\right)\right| \leq r^{O(r)}\left(\frac{\theta}{(m+n)^{2}}\right)^{(s+1) / 2}\left(\frac{\theta}{m+n}\right)^{3 r-(s+1) / 2} .
$$

Similarly, since for each isolated edge $\left(j_{1}, k_{1}\right) \in G_{0}$, variable $v_{j_{1} k_{1}}$ has to be matched with a variable $v_{j_{2} k_{2}}$ indexed by an edge $\left(j_{2}, k_{2}\right)$ in a different connected component, we conclude that each matching of the set

$$
\left\{v_{j k}:(j, k) \in G\right\}
$$

contains at least $s / 2$ weakly correlated pairs of variables and hence by (4.4)

$$
\left|\mathbf{E} t_{G}\left(v_{j k}\right)\right| \leq r^{O(r)}\left(\frac{\theta^{3}}{(m+n)^{4}}\right)^{s / 2}\left(\frac{\theta^{3}}{(m+n)^{3}}\right)^{r-s / 2} .
$$

Moreover, if $s$ is odd, the number of weakly correlated pairs in every matching is at least $(s+1) / 2$ and hence

$$
\left|\mathbf{E} t_{G}\left(v_{j k}\right)\right| \leq r^{O(r)}\left(\frac{\theta^{3}}{(m+n)^{4}}\right)^{(s+1) / 2}\left(\frac{\theta^{3}}{(m+n)^{3}}\right)^{r-(s+1) / 2}
$$

which concludes the proof of part (1).

To prove part (2), we note that a connected weighted graph $G$ of weight $e$ contains a spanning tree with at most $e$ edges and hence has at most $e+1$ vertices. In particular, a connected graph $G$ of weight $e$ contains fewer than $3 e / 2$ vertices unless $G$ is an isolated edge of weight 1 or a pair of edges of weight 1 each, sharing one common vertex. Therefore, $G$ has at most

$$
2 s+\frac{3}{2}(2 r-s)=3 r+\frac{s}{2}
$$

vertices and strictly fewer vertices, unless $s$ is even and the connected components of $G_{1}$ are pairs of edges of weight 1 each sharing one common vertex.

To prove part (3), let us define $\Sigma_{u}(G)$ as the sum in the Wick's formula over all matchings of the multiset (4.6) of the following structure: We split the edges of $G$ into $r$ pairs, pairing each isolated edge with another isolated edge and pairing each edge in a 2-edge connected component of $G$ with the remaining edge in the same connected component. We then match every variable of the multiset (4.6) with a variable indexed by an edge in the same pair. Reasoning as in part (1), we conclude that

$$
\left|\mathbf{E} t_{G}\left(u_{j k}^{3}\right)-\Sigma_{u}(G)\right| \leq \frac{r^{O(r)} \theta^{3 r}}{(m+n)^{3 r+s / 2+1}} .
$$

Similarly, let us define $\Sigma_{v}(G)$ as the sum in the Wick's formula over all matchings of the set (4.7) of the following structure: We split the edges of $G$ into $r$ pairs as above and match every variable in the set (4.7) with the variable indexed by the remaining edge of the pair. Then

$$
\left|\mathbf{E} t_{G}\left(v_{j k}\right)-\Sigma_{v}(G)\right| \leq \frac{r^{O(r)} \theta^{3 r}}{(m+n)^{3 r+s / 2+1}} .
$$


Since

$$
\Sigma_{u}(G)=\Sigma_{v}(G)
$$

the proof of part (3) follows.

Lemma 4.3. Let $u_{j k}$ be random variables as in Theorem 4.1 and let $v_{j k}$ be the auxiliary Gaussian random variables as in Section 4.2. Let

$$
U=\sum_{\substack{1 \leq j \leq m \\ 1 \leq k \leq n}} u_{j k}^{3} \text { and } V=\sum_{\substack{1 \leq j \leq m \\ 1 \leq k \leq n}} v_{j k} .
$$

Then for every integer $r>1$ we have

$$
\left|\mathbf{E} U^{2 r}-\mathbf{E} V^{2 r}\right| \leq \frac{r^{O(r)} \theta^{3 r}}{m+n} .
$$

Proof. We can write

$$
\begin{aligned}
& \mathbf{E} U^{2 r}=\sum_{G} a_{G} \mathbf{E} t_{G}\left(u_{j k}^{3}\right) \text { and } \\
& \mathbf{E} V^{2 r}=\sum_{G} a_{G} \mathbf{E} t_{G}\left(v_{j k}\right),
\end{aligned}
$$

where the sum is taken over all weighted graphs $G$ of the total weight $2 r$ and

$$
1 \leq a_{G} \leq(2 r) \text { !. }
$$

Let $\mathcal{G}_{2 r}$ be the set of weighted graphs $G$ whose connected components consist of an even number $s$ of isolated edges and $r-s / 2$ pairs of edges of weight 1 sharing one common vertex. Since there are not more than $r^{O(r)}(m+n)^{p}$ distinct weighted graphs with $p$ vertices, by parts (1) and (2) of Lemma 4.2, we conclude that

$$
\begin{aligned}
&\left|\mathbf{E} U^{2 r}-\sum_{G \in \mathcal{G}_{2 r}} a_{G} \mathbf{E} t_{G}\left(u_{j k}^{3}\right)\right| \leq \frac{r^{O(r)} \theta^{3 r}}{m+n} \text { and } \\
&\left|\mathbf{E} V^{2 r}-\sum_{G \in \mathcal{G}_{2 r}} a_{G} \mathbf{E} t_{G}\left(v_{j k}\right)\right| \leq \frac{r^{O(r)} \theta^{3 r}}{m+n} .
\end{aligned}
$$

The proof now follows by parts (1) and (3) of Lemma 4.2 .

4.4. Proof of Theorem 4.1, Let $U$ and $V$ be the random variables as in Lemma 4.3. We use the standard estimate

$$
\left|e^{i x}-\sum_{s=0}^{2 r-1} \frac{(i x)^{s}}{s !}\right| \leq \frac{x^{2 r}}{(2 r) !} \quad \text { for } \quad x \in \mathbb{R},
$$

from which it follows that

$$
\left|\mathbf{E} e^{i U}-\mathbf{E} e^{i V}\right| \leq \frac{\mathbf{E} U^{2 r}}{(2 r) !}+\frac{\mathbf{E} V^{2 r}}{(2 r) !}+\sum_{s=0}^{2 r-1} \frac{\left|\mathbf{E} U^{s}-\mathbf{E} V^{s}\right|}{s !} .
$$

By (4.5), we have

$$
\mathbf{E} U^{2}=\mathbf{E} V^{2}=O\left(\theta^{3}\right)
$$

and hence

$$
\mathbf{E} U^{2 r}, \mathbf{E} V^{2 r} \leq \frac{(2 r) !}{2^{r} r !} 2^{O(r)} \theta^{3 r}
$$


Therefore, one can choose an integer $r$ such that

$$
r \ln r \leq \gamma_{1}(\theta) \ln \frac{1}{\epsilon} \quad \text { for some constant } \quad \gamma_{1}(\theta)>0
$$

so that

$$
\frac{\mathbf{E} U^{2 r}+\mathbf{E} V^{2 r}}{(2 r) !} \leq \frac{\epsilon}{2} .
$$

By Lemma 4.3, as long as

$$
m+n \geq\left(\frac{1}{\epsilon}\right)^{\gamma(\theta)} \quad \text { for some constant } \quad \gamma(\theta)>0,
$$

we have

$$
\left|\mathbf{E} U^{2 k}-\mathbf{E} V^{2 k}\right| \leq \frac{\epsilon}{6} \quad \text { for } \quad k=0,1, \ldots, r .
$$

We note that by symmetry

$$
\mathbf{E} V^{s}=\mathbf{E} U^{s}=0 \quad \text { if } \quad s \quad \text { is odd. }
$$

Since $V$ is Gaussian and $\mathbf{E} V=0$, we have

$$
\mathbf{E} e^{i V}=\exp \left\{-\frac{1}{2} \mathbf{E} V^{2}\right\},
$$

and the proof follows by (4.8).

\section{THE FOURTH-DEGREE TERM}

In this section we prove the following main result.

Theorem 5.1. Let $w_{j k}, j=1, \ldots, m$ and $k=1, \ldots, n$ be Gaussian random variables such that

$$
\mathbf{E} w_{j k}=0 \quad \text { for all } \quad j, k .
$$

Suppose further that for some $\theta>0$ we have

$$
\begin{aligned}
& \mathbf{E} w_{j k}^{2} \leq \frac{\theta}{m+n} \quad \text { for all } j, k \text { and } \\
& \left|\mathbf{E} w_{j_{1} k_{1}} w_{j_{2} k_{2}}\right| \leq \frac{\theta}{(m+n)^{2}} \quad \text { provided } j_{1} \neq j_{1} \quad \text { and } \quad k_{1} \neq k_{2} .
\end{aligned}
$$

Let

$$
W=\sum_{\substack{1 \leq j \leq m \\ 1 \leq k \leq n}} w_{j k}^{4} .
$$

Then for some absolute constant $\gamma>0$ we have

$$
\mathbf{E} W \leq \gamma \theta^{2}
$$

$$
\operatorname{var} W \leq \frac{\gamma \theta^{4}}{m+n}
$$

$$
\mathbf{P}\left\{W>\gamma \theta^{2}+1\right\} \leq \exp \left\{-(m+n)^{1 / 5}\right\}
$$

provided $m+n \geq \gamma_{1}(\theta)$ for some $\gamma_{1}(\theta)>0$. 
We will apply Theorem 5.1 in the following situation. Let $q: \mathbb{R}^{m+n} \longrightarrow \mathbb{R}$ be the quadratic form defined by (1.5) and let $L \subset \mathbb{R}^{m+n}$ be a hyperplane not containing the null-space of $q$. Let us fix the Gaussian probability measure in $L$ with the density proportional to $e^{-q}$. We define random variables

$$
w_{j k}=\sqrt[4]{\frac{\zeta_{j k}\left(\zeta_{j k}+1\right)\left(6 \zeta_{j k}^{2}+6 \zeta_{j k}+1\right)}{24}}\left(s_{j}+t_{k}\right),
$$

where $s_{1}, \ldots, s_{m} ; t_{1}, \ldots, t_{n}$ are the coordinates of a point in $L$. Then we have

$$
W=\sum_{\substack{1 \leq k \leq m \\ 1 \leq j \leq n}} w_{j k}^{4}=h(s, t)
$$

for $h$ defined by (1.6).

While the proof of parts (1) and (2) is a straightforward computation, to prove part (3) we need reverse Hölder inequalities for polynomials with respect to the Gaussian measure.

Lemma 5.2. Let $p$ be a polynomial of degree $d$ in random Gaussian variables $w_{1}, \ldots, w_{l}$. Then for $r>2$ we have

$$
\left(\mathbf{E}|p|^{r}\right)^{1 / r} \leq r^{d / 2}\left(\mathbf{E} p^{2}\right)^{1 / 2} .
$$

Proof. This is Corollary 5 of [17].

5.1. Proof of Theorem 5.1, Using formula (4.1), we get

$$
\mathbf{E} w_{j k}^{4}=3\left(\mathbf{E} w_{j k}^{2}\right)^{2} \leq \frac{3 \theta^{2}}{(m+n)^{2}}
$$

and hence

$$
\mathbf{E} W=\sum_{\substack{1 \leq j \leq m \\ 1 \leq k \leq n}} \mathbf{E} w_{j k}^{4} \leq(m n) \frac{3 \theta^{2}}{(m+n)^{2}} \leq 3 \theta^{2},
$$

which proves part (1).

To prove part (2), we note that

$$
\operatorname{var} W=\sum_{\substack{1 \leq j_{1}, j_{2} \leq m \\ 1 \leq k_{1}, k_{2} \leq n}} \operatorname{cov}\left(w_{j_{1} k_{1}}^{4}, w_{j_{2} k_{2}}^{4}\right) .
$$

Using (4.3), we get

$$
\operatorname{cov}\left(w_{j_{1} k_{1}}^{4}, w_{j_{2} k_{2}}^{4}\right)=72\left(\mathbf{E} w_{j_{1} k_{1}} w_{j_{2} k_{2}}\right)^{2}\left(\mathbf{E} w_{j_{1} k_{1}}^{2}\right)\left(\mathbf{E} w_{j_{2} k_{2}}^{2}\right)+24\left(\mathbf{E} w_{j_{1} k_{1}} w_{j_{2} k_{2}}\right)^{4} .
$$

Hence

$$
\operatorname{cov}\left(w_{j_{1} k_{1}}^{4}, w_{j_{2} k_{2}}^{4}\right) \leq \frac{96 \theta^{4}}{(m+n)^{4}} \quad \text { for all } j_{1}, j_{2} \text { and } k_{1}, k_{2} .
$$

Additionally,

$$
\begin{aligned}
\operatorname{cov}\left(w_{j_{1} k_{1}}^{4}, w_{j_{2} k_{2}}^{4}\right) & \leq \frac{72 \theta^{4}}{(m+n)^{6}}+\frac{24 \theta^{4}}{(m+n)^{8}} \\
& \leq \frac{96 \theta^{4}}{(m+n)^{6}} \quad \text { provided } \quad j_{1} \neq j_{2} \quad \text { and } \quad k_{1} \neq k_{2} .
\end{aligned}
$$


Summarizing,

$$
\operatorname{var} W \leq m^{2} n^{2} \frac{96 \theta^{4}}{(m+n)^{6}}+\left(m n^{2}+n m^{2}\right) \frac{96 \theta^{4}}{(m+n)^{4}} \leq \frac{\gamma \theta^{4}}{m+n},
$$

which proves part (2).

To prove part (3), we apply Lemma 5.2 with $d=4$ to $p(W)=W-\mathbf{E} W$. From part (2), we get

$$
\mathbf{E}|W-\mathbf{E} W|^{r} \leq r^{2 r}(\operatorname{var} W)^{r / 2} \leq r^{2 r}\left(\frac{\gamma \theta^{4}}{m+n}\right)^{r / 2} .
$$

Let us choose

$$
r=(m+n)^{1 / 5}
$$

for sufficiently large $m+n$. Then

$$
\begin{aligned}
r^{2 r}\left(\frac{\gamma \theta^{4}}{m+n}\right)^{r / 2} & =\exp \left\{2 r \ln r+\frac{r}{2} \ln \left(\gamma \theta^{4}\right)-\frac{r}{2} \ln (m+n)\right\} \\
& =\exp \left\{-\frac{1}{10}(m+n)^{1 / 5} \ln (m+n)+\frac{\ln \left(\gamma \theta^{4}\right)}{2}(m+n)^{1 / 5}\right\} \\
& \leq \exp \left\{-\frac{(m+n)^{1 / 5}}{10}\right\}
\end{aligned}
$$

provided $\ln (m+n)>5 \ln \left(\gamma \theta^{4}\right)+10$.

Hence if $m+n$ is sufficiently large, we have

$$
\mathbf{E}|W-\mathbf{E} W|^{r} \leq \exp \left\{-\frac{(m+n)^{1 / 5}}{10}\right\} .
$$

The proof follows by part (1) and Markov's inequality.

\section{Computing the integral over a Neighborhood of the ORIGiN}

We consider the integral

$$
\int_{\Pi_{0}} F(s, t) d s d t
$$

of Corollary 2.2. Recall that $\Pi_{0}$ is the facet of the parallelepiped

$$
\Pi=\left\{\left(s_{1}, \ldots, s_{m} ; t_{1}, \ldots, t_{n}\right): \quad-\pi \leq s_{j}, t_{k} \leq \pi \text { for all } j, k\right\}
$$

defined by the equation $t_{n}=0$ and that

$$
F(s, t)=\exp \left\{-i \sum_{j=1}^{m} r_{j} s_{j}-i \sum_{k=1}^{n} c_{k} t_{k}\right\} \prod_{\substack{1 \leq j \leq m \\ 1 \leq k \leq n}} \frac{1}{1+\zeta_{j k}-\zeta_{j k} e^{i\left(s_{j}+t_{k}\right)}} .
$$

In this section, we prove the following main result.

Theorem 6.1. Let us fix a number $0<\delta<1$. Suppose that $m \geq \delta n, n \geq \delta m$ and that

$$
\delta \tau \leq \zeta_{j k} \leq \tau \text { for all } j, k
$$

and some $\tau>\delta$. 
Let $q: \mathbb{R}^{m+n} \longrightarrow \mathbb{R}$ be the quadratic form defined by (1.5) and let $f, h$ : $\mathbb{R}^{m+n} \longrightarrow \mathbb{R}$ be the polynomials defined by (1.6). Let us define a neighborhood $\mathcal{U} \subset \Pi_{0}$ by

$$
\mathcal{U}=\left\{(s, t) \in \Pi_{0}: \quad\left|s_{j}\right|,\left|t_{k}\right| \leq \frac{\ln (m+n)}{\tau \sqrt{m+n}} \text { for all } j, k\right\} .
$$

Let us identify the hyperplane $t_{n}=0$ containing $\Pi_{0}$ with $\mathbb{R}^{m+n-1}$, let

$$
\Xi=\int_{\mathbb{R}^{m+n-1}} e^{-q} d s d t
$$

and let us consider the Gaussian probability measure in $\mathbb{R}^{m+n-1}$ with the density $\Xi^{-1} e^{-q}$. Let

$$
\mu=\mathbf{E} f^{2} \quad \text { and } \quad \nu=\mathbf{E} h .
$$

Then

(1)

$$
\Xi \geq \frac{(2 \pi)^{(m+n-1) / 2}}{m^{(n-1) / 2} n^{(m-1) / 2}\left(\tau+\tau^{2}\right)^{(m+n-1) / 2}} .
$$

$(2)$

$$
0 \leq \mu, \nu \leq \gamma(\delta)
$$

for some constant $\gamma(\delta)>0$.

(3) For any $0<\epsilon \leq 1 / 2$

$$
\left|\int_{\mathcal{U}} F(s, t) d s d t-\exp \left\{-\frac{\mu}{2}+\nu\right\} \Xi\right| \leq \epsilon \Xi
$$

provided

$$
m+n \geq\left(\frac{1}{\epsilon}\right)^{\gamma(\delta)}
$$

for some constant $\gamma(\delta)>0$.

(4) For any $0 \leq \epsilon \leq 1 / 2$

$$
\left|\int_{\mathcal{U}}\right| F(s, t)|d s d t-\exp \{\nu\} \Xi| \leq \epsilon \Xi
$$

provided

$$
m+n \geq\left(\frac{1}{\epsilon}\right)^{\gamma(\delta)}
$$

for some constant $\gamma(\delta)>0$.

Proof. All implied constants in the " $O$ " and " $\Omega$ " notation below may depend only on the parameter $\delta$.

Let

$$
q_{0}(s, t)=\frac{1}{2} \sum_{\substack{1 \leq j \leq m \\ 1 \leq k \leq n}}\left(s_{j}+t_{k}\right)^{2}
$$

as in Lemma 3.5. Then

$$
q(s, t) \leq\left(\tau+\tau^{2}\right) q_{0}(s, t)
$$


and, therefore,

$$
\begin{aligned}
\int_{\mathbb{R}^{m+n-1}} \exp \{-q(s, t)\} d s d t & \geq \int_{\mathbb{R}^{m+n-1}} \exp \left\{-\left(\tau+\tau^{2}\right) q_{0}(s, t)\right\} d s d t \\
& =\frac{\pi^{(m+n-1) / 2}}{\left(\tau^{2}+\tau\right)^{(m+n-1) / 2} \sqrt{\operatorname{det} q_{0} \mid \mathbb{R}^{m+n-1}}},
\end{aligned}
$$

where $q_{0} \mid \mathbb{R}^{m+n-1}$ is the restriction of the form $q_{0}$ onto the coordinate hyperplane $t_{n}=0$ in $\mathbb{R}^{m+n}$.

Let $H \subset \mathbb{R}^{m+n}$ be the orthogonal complement complement in $\mathbb{R}^{m+n}$ to the kernel of $q_{0}$, that is, the hyperplane defined by the equation:

$$
s_{1}+\ldots+s_{m}=t_{1}+\ldots+t_{n} \quad \text { for } \quad(s, t)=\left(s_{1}, \ldots, s_{m} ; t_{1}, \ldots, t_{n}\right) .
$$

Then, from the eigenspace decomposition of Lemma 3.5 it follows that

$$
\operatorname{det} q_{0} \mid H=\left(\frac{m}{2}\right)^{n-1}\left(\frac{n}{2}\right)^{m-1}\left(\frac{m+n}{2}\right) .
$$

On the other hand, by Lemma 3.4

$$
\operatorname{det} q_{0}\left|\mathbb{R}^{m+n-1}=\frac{1}{m+n} \operatorname{det} q_{0}\right| H
$$

and the proof of part (1) follows.

Let us consider the coordinate functions $s_{j}, t_{k}$ as random variables on the space $\mathbb{R}^{m+n-1}$ with the Gaussian probability measure with the density $\Xi^{-1} e^{-q}$. From Theorem 3.2 ,

$$
\mathbf{E}\left(s_{j}+t_{k}\right)^{2}=O\left(\frac{1}{\tau^{2}(m+n)}\right) \quad \text { for all } j, k
$$

and

$$
\begin{gathered}
\left|\mathbf{E}\left(s_{j_{1}}+t_{k_{1}}\right)\left(s_{j_{2}}+t_{k_{2}}\right)\right|=O\left(\frac{1}{\tau^{2} m n}\right) \\
\text { if } j_{1} \neq j_{2} \quad \text { and } \quad k_{1} \neq k_{2} .
\end{gathered}
$$

Let

$$
\begin{aligned}
u_{j k} & =\sqrt[3]{\frac{\zeta_{j k}\left(\zeta_{j k}+1\right)\left(2 \zeta_{j k}+1\right)}{6}}\left(s_{j}+t_{k}\right) \\
w_{j k} & =\sqrt[4]{\frac{\zeta_{j k}\left(\zeta_{j k}+1\right)\left(6 \zeta_{j k}^{2}+6 \zeta_{j k}+1\right)}{24}}\left(s_{j}+t_{k}\right) \text { for all } j, k .
\end{aligned}
$$

Then $u_{j k}$ and $w_{j k}$ are Gaussian random variables such that

$$
\begin{aligned}
\mathbf{E} u_{j k}^{2}, \mathbf{E} w_{j k}^{2}= & O\left(\frac{1}{m+n}\right) \quad \text { for all } j, k \\
& \text { and } \\
\left|\mathbf{E} u_{j_{1} k_{1}} u_{j_{2} k_{2}}\right|,\left|\mathbf{E} w_{j_{1} k_{1}} w_{j_{2} k_{2}}\right|= & O\left(\frac{1}{m n}\right) \\
& \text { if } \quad j_{1} \neq j_{2} \text { and } k_{1} \neq k_{2} .
\end{aligned}
$$


We observe that

$$
f=\sum_{\substack{1 \leq j \leq m \\ 1 \leq k \leq n}} u_{j k}^{3} \quad \text { and } \quad h=\sum_{\substack{1 \leq j \leq m \\ 1 \leq k \leq n}} w_{j k}^{4} .
$$

Therefore, the bound

$$
\mu=\mathbf{E} f^{2}=O(1)
$$

follows by Theorem 4.1 while the bound

$$
\nu=\mathbf{E} h=O(1)
$$

follows by part (1) of Theorem 5.1. This concludes the proof of part (2).

Since $s_{j}+t_{k}$ is a Gaussian random variable, by the first inequality of (6.1), we conclude that

$$
\mathbf{P}\left\{\left|s_{j}+t_{k}\right| \geq \frac{\ln (m+n)}{4 \tau \sqrt{m+n}}\right\} \leq \exp \left\{-\Omega\left(\ln ^{2}(m+n)\right)\right\} .
$$

Note that the inequalities hold for $k=n$ with $t_{n}=0$ as well, and hence

$$
\int_{\mathbb{R}^{m+n-1} \backslash \mathcal{U}} e^{-q} d s d t \leq \exp \left\{-\Omega\left(\ln ^{2}(m+n)\right)\right\} \Xi,
$$

where $\mathcal{U}$ is the neighborhood defined in the theorem. Therefore,

$$
\left|\int_{\mathbb{R}^{m+n-1} \backslash \mathcal{U}} \exp \{-q-i f\} d s d t\right| \leq \exp \left\{-\Omega\left(\ln ^{2}(m+n)\right)\right\} \Xi .
$$

Since $f=\sum_{j k} u_{j k}^{3}$ and Gaussian random variables $u_{j k}$ satisfy (6.2), from Theorem 4.1 we conclude that for any $0 \leq \epsilon \leq 1 / 2$ we have

$$
\begin{gathered}
\left|\int_{\mathbb{R}^{m+n-1}} \exp \{-q-i f\} d s d t-\exp \left\{-\frac{\mu}{2}\right\}\right| \leq \epsilon \Xi \\
\text { provided } m+n>\left(\frac{1}{\epsilon}\right)^{O(1)} .
\end{gathered}
$$

Therefore, for any $0 \leq \epsilon \leq 1 / 2$ we have

$$
\begin{gathered}
\left|\int_{\mathcal{U}} \exp \{-q-i f\} d s d t-\exp \left\{-\frac{\mu}{2}\right\}\right| \leq \epsilon \Xi \\
\text { provided } m+n>\left(\frac{1}{\epsilon}\right)^{O(1)} .
\end{gathered}
$$

Since $h=\sum_{j k} w_{j k}^{4}$ and Gaussian random variables $w_{j k}$ satisfy ([6.2) , by part (2) of Theorem [5.1] we have

$$
\operatorname{var} h=\mathbf{E}(h-\nu)^{2}=O\left(\frac{1}{m+n}\right) .
$$

Applying Chebyshev's inequality, we conclude that for any $\epsilon>0$

$$
\int_{\substack{(s, t) \in \mathcal{U}: \\|h(s, t)-\nu| \geq \epsilon}} e^{-q} d s d t=O\left(\frac{1}{\epsilon^{2}(m+n)}\right) \Xi .
$$


By part (3) of Theorem [5.1, for some constant $\gamma(\delta)>0$ we have

$$
\int_{\substack{(s, t) \in \mathcal{U}: \\ h(s, t) \geq \gamma(\delta)}} e^{-q} d s d t=O\left(\exp \left\{-(m+n)^{1 / 5}\right\}\right) \Xi .
$$

In addition,

$$
h=O\left(\ln ^{4}(m+n)\right) \text { in } \mathcal{U} .
$$

In view of (6.3) and part (2) of the theorem, (6.5) we have

$$
\begin{gathered}
\left|\int_{\mathcal{U}} \exp \{-q+h\} d s d t-\exp \{\nu\}\right| \leq \epsilon \Xi \\
\text { provided } m+n>\left(\frac{1}{\epsilon}\right)^{O(1)} .
\end{gathered}
$$

Similarly, from (6.4) - 6.7) we deduce that

$$
\begin{aligned}
& \left|\int_{\mathcal{U}} \exp \{-q-i f+h\} d s d t-\exp \left\{-\frac{\mu}{2}+\nu\right\}\right| \leq \epsilon \Xi \\
& \quad \text { provided } m+n>\left(\frac{1}{\epsilon}\right)^{O(1)} .
\end{aligned}
$$

From the Taylor series expansion, cf. (2.2), we write

$$
\begin{gathered}
F(s, t)=\exp \{-q(s, t)-i f(s, t)+h(s, t)+\rho(s, t)\} \quad \text { where } \\
|\rho(s, t)|=O\left(\frac{\ln ^{5}(m+n)}{\sqrt{m+n}}\right) \quad \text { for } \quad(s, t) \in \mathcal{U} .
\end{gathered}
$$

Therefore, using (6.8) and part (2) of the theorem we conclude that

$$
\begin{aligned}
& \left|\int_{\mathcal{U}} F d s d t-\int_{\mathcal{U}} \exp \{-q-i f+h\} d s d t\right|=O\left(\frac{\ln ^{5}(m+n)}{\sqrt{m+n}}\right) \Xi \\
& \left|\int_{\mathcal{U}}\right| F\left|d s d t-\int_{\mathcal{U}} \exp \{-q+h\} d s d t\right|=O\left(\frac{\ln ^{5}(m+n)}{\sqrt{m+n}}\right) \Xi .
\end{aligned}
$$

We complete the proof of parts (3) and (4) from (6.8) and (6.9).

\section{Bounding THE INTEGRAL OUtSide OF A NEIGHBORHOOD OF THE ORIGIN}

We consider the integral representation of Corollary 2.2. In this section we prove that the integral of $F(s, t)$ outside of the neighborhood $\mathcal{U}$ of the origin is asymptotically negligible (note that by Theorem 6.1 the integral of $F$ and the integral of $|F|$ over $\mathcal{U}$ have the same order). We prove the following main result.

Theorem 7.1. Let us fix a number $0<\delta<1$. Suppose that $m \geq \delta n, n \geq \delta m$ and that

$$
\delta \tau \leq \zeta_{j k} \leq \tau \text { for all } j, k
$$

and some $\delta \leq \tau \leq(m+n)^{1 / \delta}$. Let

$$
\mathcal{U}=\left\{(s, t) \in \Pi_{0}: \quad\left|s_{j}\right|,\left|t_{k}\right| \leq \frac{\ln (m+n)}{\tau \sqrt{m+n}} \text { for all } j, k\right\} .
$$


Then for any $\kappa>0$

$$
\int_{\Pi_{0} \backslash \mathcal{U}}|F(s, t)| d s d t \leq(m+n)^{-\kappa} \int_{\Pi_{0}}|F(s, t)| d s d t
$$

provided $m+n>\gamma(\delta, \kappa)$ for some $\gamma(\delta, \kappa)>0$.

We prove Theorem 7.1 it in two steps. First, by a string of combinatorial arguments we show that the integral

$$
\int_{\Pi_{0} \backslash I}|F(s, t)| d s d t
$$

is negligible compared to

$$
\int_{I}|F(s, t)| d s d t
$$

where $I \subset \Pi_{0}$ is a larger neighborhood of the origin

$$
I=\left\{(s, t) \in \Pi_{0}: \quad\left|s_{j}\right|,\left|t_{k}\right| \leq \epsilon / \tau \quad \text { for all } j, k\right\}
$$

and $\epsilon>0$ is any fixed number. This is the only place where we use that $\tau$ is bounded above by a polynomial in $m+n$. Then we notice that for a sufficiently small $\epsilon=\epsilon(\delta)$, the function $|F(s, t)|$ is strictly log-concave on $I$ and we use a concentration inequality for strictly log-concave measures to deduce that the integral

$$
\int_{I \backslash \mathcal{U}}|F(s, t)| d s d t
$$

is negligible compared to (7.1).

7.1. Metric $\rho$. Let us introduce the following function

$$
\rho: \mathbb{R} \longrightarrow[0, \pi], \quad \rho(x)=\min _{k \in \mathbb{Z}}|x-2 \pi k| .
$$

In words: $\rho(x)$ is the distance from $x$ to the closest integer multiple of $2 \pi$. Clearly,

$$
\rho(-x)=\rho(x) \quad \text { and } \quad \rho(x+y) \leq \rho(x)+\rho(y)
$$

for all $x, y \in \mathbb{R}$. We will use that

$$
1-\frac{1}{2} \rho^{2}(x) \leq \cos x \leq 1-\frac{1}{5} \rho^{2}(x) .
$$

7.2. The absolute value of $F(s, t)$. Let

$$
\alpha_{j k}=2 \zeta_{j k}\left(1+\zeta_{j k}\right) \quad \text { for all } j, k .
$$

Then

$$
2 \delta^{2} \tau^{2} \leq \alpha_{j k} \leq 4 \tau^{2} \text { for all } j, k .
$$

Let us define functions

$$
f_{j k}(x)=\frac{1}{\sqrt{1+\alpha_{j k}-\alpha_{j k} \cos x}} \quad \text { for } \quad x \in \mathbb{R} .
$$

Then we can write

$$
|F(s, t)|=\prod_{\substack{1 \leq j \leq m \\ 1 \leq k \leq n}} f_{j k}\left(s_{j}+t_{k}\right) .
$$


We observe that

$$
f_{j k}(x)=1 \quad \text { provided } \quad \rho(x)=0 \text { and that } f_{j k}(-x)=f_{j k}(x) .
$$

From (7.2) and (7.3) we conclude that for any $\epsilon>0$, we have

$$
\begin{aligned}
f_{j k}(x) \leq & \exp \{-\gamma(\delta, \epsilon)\} f_{j k}(y) \\
& \text { provided } \rho(x) \geq \frac{2 \epsilon}{\tau} \text { and } \rho(y) \leq \frac{\epsilon}{\tau},
\end{aligned}
$$

where $\gamma(\delta, \epsilon)>0$ is a constant.

Finally, we observe that

$$
\frac{d^{2}}{d x^{2}} \ln f_{j k}(x)=-\frac{\alpha_{j k}\left(1+\alpha_{j k}\right) \cos x-\alpha_{j k}^{2}}{2\left(1+\alpha_{j k}-\alpha_{j k} \cos x\right)^{2}} .
$$

It follows from (7.2) and (7.3) that for all $j, k$

$$
\frac{d^{2}}{d x^{2}} \ln f_{j k}(x) \leq-\frac{2}{5} \tau^{2} \delta^{2} \quad \text { provided } \quad|x| \leq \frac{\delta^{2}}{5 \tau} .
$$

In particular, the function $\ln f_{j k}(x)$ is strictly log-concave on the interval $|x| \leq$ $\delta^{2} / 5 \tau$.

In what follows, we fix a particular parameter $\epsilon>0$. All implied constants in the " $O$ " and " $\Omega$ " notation may depend only on the parameters $\delta$ and $\epsilon$. We say that $m$ and $n$ are sufficiently large if $m+n \geq \gamma(\delta, \epsilon)$ for some constant $\gamma(\delta, \epsilon)>0$. Recall that $m$ and $n$ are of the same order, $m \geq \delta n$ an $n \geq \delta m$.

Our first goal is to show that for any fixed $\epsilon>0$ only the points $(s, t) \in \Pi_{0}$ for which the inequality $\rho\left(s_{j}+t_{k}\right) \leq \epsilon / \tau$ holds for the the overwhelming majority of pairs of indices $(j, k)$ contribute significantly to the integral of $|F(s, t)|$ on $\Pi_{0}$. Recall that $\tau_{n}=0$ on $\Pi_{0}$.

Lemma 7.2. For $\epsilon>0$ and a point $(s, t) \in \Pi_{0}$ let us define the following two sets:

Let $J=J(s, t ; \epsilon) \subset\{1, \ldots, m\}$ be the set of all indices $j$ such that

$$
\rho\left(s_{j}+t_{k}\right) \leq \epsilon / \tau
$$

for more than $(n-1) / 2$ distinct indices $k=1, \ldots, n-1$.

Let $K=K(s, t ; \epsilon) \subset\{1, \ldots, n-1\}$ be the set of all indices $k$ such that

$$
\rho\left(s_{j}+t_{k}\right) \leq \epsilon / \tau
$$

for more than $m / 2$ distinct indices $j=1, \ldots, m$.

Let $\bar{J}=\{1, \ldots, m\} \backslash J$ and let $\bar{K}=\{1, \ldots, n-1\} \backslash K$.

Then

(1)

$$
\begin{aligned}
|F(s, t)| & \leq \exp \{-\gamma(\delta, \epsilon) n|\bar{J}|\} \quad \text { and } \\
|F(s, t)| & \leq \exp \{-\gamma(\delta, \epsilon) m|\bar{K}|\}
\end{aligned}
$$

for some constant $\gamma(\delta, \epsilon)>0$.

$$
\begin{aligned}
& \rho\left(s_{j_{1}}-s_{j_{2}}\right) \leq 2 \epsilon / \tau \text { for all } j_{1}, j_{2} \in J \quad \text { and } \\
& \rho\left(t_{k_{1}}-t_{k_{2}}\right) \leq 2 \epsilon / \tau \text { for all } k_{1}, k_{2} \in K . \\
&(3) \text { If }|J|>m / 2 \text { or }|K|>(n-1) / 2 \text {, then } \\
& \rho\left(s_{j}+t_{k}\right) \leq 3 \epsilon / \tau \text { for all } j \in J \text { and all } k \in K .
\end{aligned}
$$


Proof. For every $j \in \bar{J}$ there are at least $(n-1) / 2$ distinct $k$ and for every $k \in \bar{K}$ there are at least $m / 2$ distinct $j$ such that

$$
\rho\left(s_{j}+t_{k}\right)>\epsilon / \tau
$$

and hence

$$
f_{j k}\left(s_{j}+t_{k}\right) \leq \exp \{-\Omega(1)\}
$$

by (7.5). Part (1) follows from (7.4).

For every $j_{1}, j_{2} \in J$ there is at least one common index $k$ such that

$$
\rho\left(s_{j_{1}}+t_{k}\right) \leq \epsilon / \tau \text { and } \rho\left(s_{j_{2}}+t_{k}\right) \leq \epsilon / \tau .
$$

Then

$$
\rho\left(s_{j_{1}}-s_{j_{2}}\right)=\rho\left(s_{j_{1}}+t_{k}-s_{j_{2}}-t_{k}\right) \leq \rho\left(s_{j_{1}}+t_{k}\right)+\rho\left(s_{j_{2}}+t_{k}\right) \leq 2 \epsilon / \tau .
$$

The second inequality of part (2) follows similarly.

If $|K|>(n-1) / 2$, then for every $j \in J$ there is a $k_{j} \in K$ such that

$$
\rho\left(s_{j}+t_{k_{j}}\right) \leq \epsilon / \tau \text {. }
$$

Then, by part (2), for every $k \in K$ we have

$$
\rho\left(s_{j}+t_{k}\right)=\rho\left(s_{j}+t_{k_{j}}-t_{k_{j}}+t_{k}\right) \leq \rho\left(s_{j}+t_{k_{j}}\right)+\rho\left(t_{k}-t_{k_{j}}\right) \leq 3 \epsilon / \tau .
$$

The case of $|J|>m / 2$ is handled similarly.

Using estimates of Theorem 6.1 it is not hard to deduce from part (1) of Lemma 7.2 that for any fixed $\epsilon>0$ only points $(s, t) \in \Pi_{0}$ with $\bar{J}=O(\ln m)$ and $\bar{K}=$ $O(\ln n)$ may contribute essentially to the integral of $|F(s, t)|$. It follows then by part (3) of Lemma 7.2 that for such points we have $\rho\left(s_{j}+t_{k}\right) \leq 3 \epsilon / \tau$ for all $j \in J$ and all $k \in K$. Our next goal is to show that only those points $(s, t) \in \Pi_{0}$ contribute substantially to the integral for which $\rho\left(s_{j}+t_{k}\right) \leq \epsilon / \tau$ for all pairs $(j, k)$.

Proposition 7.3. For $\epsilon>0$ let us define a set $X(\epsilon) \subset \Pi_{0}$ by

$$
\begin{aligned}
X(\epsilon)=\left\{(s, t) \in \Pi_{0}:\right. & \rho\left(s_{j}+t_{k}\right) \leq \epsilon / \tau \\
& \text { for all } j=1, \ldots, m \text { and } k=1, \ldots, n-1\} .
\end{aligned}
$$

Then

$$
\int_{\Pi_{0} \backslash X(\epsilon)}|F(s, t)| d s d t \leq \exp \{-\gamma(\delta, \epsilon)(m+n)\} \int_{\Pi_{0}}|F(s, t)| d s d t
$$

for some constant $\gamma(\delta, \epsilon)>0$ and all sufficiently large $m+n$.

Proof. For subsets $A \subset\{1, \ldots, m\}$ and $B \subset\{1, \ldots, n-1\}$ let us define a set $P_{A, B} \subset \Pi_{0}$ (we call it a piece) by

$$
P_{A, B}=\left\{(s, t) \in \Pi_{0}: \quad \rho\left(s_{j}+t_{k}\right) \leq \epsilon / 40 \tau \text { for all } j \in A \text { and all } k \in B\right\} .
$$

Let

$$
V=\bigcup_{A, B} P_{A, B}
$$

where the union is taken over all subsets $A$ and $B$ such that

$$
|\bar{A}| \leq \ln ^{2} m \quad \text { and } \quad \bar{B} \leq \ln ^{2} n
$$

where

$$
\bar{A}=\{1, \ldots, m\} \backslash A \text { and } \bar{B}=\{1, \ldots, n-1\} \backslash B .
$$


We claim that the integral over $\Pi_{0} \backslash V$ is asymptotically negligible. Indeed, for sufficiently large $m+n$ and for all $(s, t) \in \Pi \backslash V$, by part (3) of Lemma 7.2, we must have

$$
\bar{J}(s, t ; \epsilon / 120) \geq \ln ^{2} m \quad \text { or } \quad \bar{K}(s, t ; \epsilon / 120) \geq \ln ^{2} n .
$$

In either case, by part (1) of Lemma 7.2, we must have

$$
|F(s, t)| \leq \exp \left\{-\Omega\left(n \ln ^{2} n\right)\right\}
$$

for all sufficiently large $m+n$. On the other hand, by parts (1), (2) and (4) of Theorem 6.1, we conclude that

$$
\int_{\Pi_{0}}|F(s, t)| d s d t \geq \exp \{-O(n \ln n)\}
$$

(we use that $\tau$ is bounded by a polynomial in $m+n$ ). This proves that

$$
\int_{\Pi_{0} \backslash V}|F(s, t)| d s d t \leq \exp \left\{-\Omega\left(n \ln ^{2} n\right)\right\} \int_{\Pi_{0}}|F(s, t)| d s d t
$$

provided $m+n$ is sufficiently large.

Next, we prove that the integral over $V \backslash X(\epsilon)$ is asymptotically negligible.

As in the proof of part (2) of Lemma 7.2 we conclude that for every piece $P_{A, B}$ and for every $(s, t) \in P_{A, B}$ we have

$$
\begin{aligned}
& \rho\left(s_{j_{1}}-s_{j_{2}}\right) \leq \epsilon / 20 \tau \text { for all } j_{1}, j_{2} \in A \\
& \rho\left(t_{k_{1}}-t_{k_{2}}\right) \leq \epsilon / 20 \tau \text { for all } k_{1}, k_{2} \in B .
\end{aligned} \quad \text { and }
$$

Let us choose a point $(s, t) \in P_{A, B} \backslash X(\epsilon)$. Hence we have $\rho\left(s_{j_{0}}+t_{k_{0}}\right)>\epsilon / \tau$ for some $j_{0}$ and $k_{0}$. Let us pick any $j_{1} \in A$ and $k_{1} \in B$. Then

$$
\begin{aligned}
\rho\left(s_{j_{0}}+t_{k_{0}}\right) & =\rho\left(s_{j_{0}}+t_{k_{0}}+s_{j_{1}}+t_{k_{1}}-s_{j_{1}}-t_{k_{1}}\right) \\
& \leq \rho\left(s_{j_{0}}+t_{k_{1}}\right)+\rho\left(s_{j_{1}}+t_{k_{0}}\right)+\rho\left(s_{j_{1}}+t_{k_{1}}\right) .
\end{aligned}
$$

Since $\rho\left(s_{j_{1}}+t_{k_{1}}\right) \leq \epsilon / 40 \tau$, we must have either

$$
\rho\left(s_{j_{0}}+t_{k_{1}}\right)>39 \epsilon / 80 \tau,
$$

in which case necessarily $j_{0} \in \bar{A}$, or

$$
\rho\left(s_{j_{1}}+t_{k_{0}}\right)>39 \epsilon / 80 \tau,
$$

in which case necessarily $k_{0} \in \bar{B}$.

In the first case (7.8) implies that

$$
\rho\left(s_{j_{0}}+t_{k}\right)>35 \epsilon / 80 \tau=7 \epsilon / 16 \tau \text { for all } k \in B
$$

and in the second case (7.8) implies that

$$
\rho\left(s_{j}+t_{k_{0}}\right)>35 \epsilon / 80 \tau=7 \epsilon / 16 \tau \text { for all } j \in A .
$$

For $j \in \bar{A}$ we define

$$
Q_{A, B ; j}=\left\{(s, t) \in P_{A, B}: \quad \rho\left(s_{j}+t_{k}\right)>7 \epsilon / 16 \tau \text { for all } k \in B\right\}
$$

and for $k \in \bar{B}$ we define

$$
R_{A, B ; k}=\left\{(s, t) \in P_{A, B}: \quad \rho\left(s_{j}+t_{k}\right)>7 \epsilon / 16 \tau \text { for all } j \in A\right\} .
$$


Then

$$
P_{A, B} \backslash X(\epsilon) \subset\left(\bigcup_{j \in \bar{A}} Q_{A, B ; j} \quad \bigcup \bigcup_{k \in \bar{B}} R_{A, B ; k}\right) .
$$

Let us compare the integrals

$$
\int_{Q_{A, B ; j}}|F(s, t)| d s d t \text { and } \int_{P_{A, B}}|F(s, t)| d s d t .
$$

Given a point $(s, t) \in P_{A, B}$ we obtain another point in $P_{A, B}$ if we arbitrarily choose coordinates $s_{j} \in[-\pi, \pi]$ for $j \in \bar{A}$ and $t_{k} \in[-\pi, \pi]$ for $k \in \bar{B}$. Let us pick a particular non-empty set $Q_{A, B ; j_{0}}$ for some $j_{0} \in \bar{A}$. We obtain a fiber $E=E_{j_{0}} \subset$ $P_{A, B}$ if we let the coordinate $s_{j_{0}}$ vary arbitrarily between $-\pi$ and $\pi$ while fixing all other coordinates of some point $(s, t) \in P_{A, B}$. Geometrically, each fiber $E$ is an interval of length $2 \pi$. We construct a set $I \subset E$ as follows: We choose an arbitrary coordinate $k_{1} \in B$ and let $s_{j_{0}}$ vary in such a way that $\rho\left(s_{j_{0}}+t_{k_{1}}\right) \leq \epsilon / 20 \tau$. Geometrically, $I$ is an interval of length $\epsilon / 10 \tau$ or a union of two non-overlapping intervals of the total length $\epsilon / 10 \tau$. Moreover, by (7.8), we have

$$
\rho\left(s_{j_{0}}+t_{k}\right) \leq \epsilon / 10 \tau \text { for all } k \in B \text { and all }(s, t) \in I .
$$

As we vary $s_{j_{0}}$ without changing other coordinates, in the product (7.4) only the functions $f_{j_{0} k}$ change. Comparing (7.12) and (7.9) and using (7.4) and (7.5), we conclude that

$$
\begin{aligned}
& |F(s, t)| \leq \exp \{-\Omega(n)\}|F(\tilde{s}, \tilde{t})| \\
& \quad \text { for all } \quad(s, t) \in Q_{A, B ; j_{0}} \cap E \text { and all }(\tilde{s}, \tilde{t}) \in I .
\end{aligned}
$$

Therefore,

$$
\int_{E \cap Q_{A, B ; j_{0}}}|F(s, t)| d s_{j_{0}} \leq \exp \{-\Omega(n)\} \int_{E}|F(s, t)| d s_{j_{0}}
$$

provided $m+n$ is large enough (again, we use that $\tau$ is bounded by a polynomial in $m+n)$. Integrating over all fibers $E \subset P_{A, B}$, we prove that

$$
\int_{Q_{A, B ; j}}|F(s, t)| d s d t \leq \exp \{-\Omega(n)\} \int_{P_{A, B}}|F(s, t)| d s d t
$$

provided $m+n$ is large enough. Similarly, we prove that for sets $R_{A, B ; k}$ defined by (17.10) we have

$$
\int_{R_{A, B ; k}}|F(s, t)| d s d t \leq \exp \{-\Omega(n)\} \int_{P_{A, B}}|F(s, t)| d s d t
$$

provided $m+n$ is large enough. Since $|\bar{A}| \leq \ln ^{2} m$ and $\bar{B} \mid \leq \ln ^{2} n$, from (7.11) we deduce that

$$
\int_{P_{A, B} \backslash X(\epsilon)}|F(s, t)| d s d t \leq \exp \{-\Omega(n)\} \int_{P_{A, B}}|F(s, t)| d s d t
$$

provided $m+n$ is large enough. Finally, since the number of pieces $P_{A, B}$ does not exceed $\exp \left\{O\left(\ln ^{3} n\right)\right\}$, the proof follows by (7.7). 
Our next goal is to show that the integral over $\Pi_{0} \backslash I$ is negligible, where

$$
I=\left\{(s, t) \in \Pi_{0}: \quad\left|s_{j}\right|,\left|t_{k}\right| \leq \epsilon / \tau \text { for all } j, k\right\} .
$$

We accomplish this in the next two lemmas.

Lemma 7.4. For $\epsilon>0$ let us define a set $Y(\epsilon) \subset \Pi_{0}$ by

$$
\begin{aligned}
Y(\epsilon)=\left\{(s, t) \in \Pi_{0}:\right. & \left|s_{j}+t_{k}\right| \leq \epsilon / \tau \\
& \text { for all } j=1, \ldots, m \text { and all } k=1, \ldots, n-1\} .
\end{aligned}
$$

Then

$$
\int_{\Pi_{0} \backslash Y(\epsilon)}|F(s, t)| d s d t \leq \exp \{-\gamma(\delta, \epsilon)(m+n)\} \int_{\Pi_{0}}|F(s, t)| d s d t,
$$

for some constant $\gamma(\epsilon, \delta)>0$ and all sufficiently large $m+n$.

Proof. Without loss of generality, we assume that $\epsilon<\delta / 2$, so $\epsilon / \tau<1 / 2$.

Let $X(\epsilon)$ be the set of Proposition 7.3 and let us define

$$
\begin{array}{r}
Z(\epsilon)=\left\{(s, t) \in \Pi_{0}: \quad s_{j}, t_{k} \in[-\pi,-\pi+\epsilon / \tau] \cup[\pi-\epsilon / \tau, \pi]\right. \\
\text { for all } j=1, \ldots, m \quad \text { and all } k=1, \ldots, n-1\} .
\end{array}
$$

We claim that

$$
X(\epsilon) \subset Y(\epsilon) \cup Z(2 \epsilon) .
$$

We note that if $\rho(x) \leq \epsilon / \tau$ for some $-2 \pi \leq x \leq 2 \pi$, then either $|x| \leq \epsilon / \tau$ or $x \geq 2 \pi-\epsilon / \tau$ or $x \leq-2 \pi+\epsilon / \tau$. To prove (7.13), let us pick an arbitrary $(s, t) \in X(\epsilon)$. Suppose that

$$
-\pi+2 \epsilon / \tau<s_{j_{0}}<\pi-2 \epsilon / \tau \text { for some } j_{0} .
$$

Since $-\pi \leq t_{k} \leq \pi$ for all $k$, we have

$$
-2 \pi+2 \epsilon / \tau<s_{j_{0}}+t_{k}<2 \pi-2 \epsilon / \tau \text { for } k=1, \ldots, n .
$$

Since $\rho\left(s_{j_{0}}+t_{k}\right) \leq \epsilon / \tau$, we must have

$$
\left|s_{j_{0}}+t_{k}\right| \leq \epsilon / \tau \quad \text { for } \quad k=1, \ldots, n-1
$$

and, therefore,

$$
-\pi+\epsilon / \tau<t_{k}<\pi-\epsilon / \tau \text { for } \quad k=1, \ldots, n-1 .
$$

Since $-\pi \leq s_{j} \leq \pi$, we conclude that

$$
\begin{aligned}
& -2 \pi+\epsilon / \tau<s_{j}+t_{k}<2 \pi-\epsilon / \tau \\
& \quad \text { for all } j=1, \ldots, m \text { and } k=1, \ldots, n-1 .
\end{aligned}
$$

Since $\rho\left(s_{j}+t_{k}\right) \leq \epsilon / \tau$, we conclude that

$$
\begin{aligned}
& \left|s_{j}+t_{k}\right| \leq \epsilon / \tau \\
& \quad \text { for all } j=1, \ldots, m \text { and } k=1, \ldots, n-1
\end{aligned}
$$

and hence $(s, t) \in Y(\epsilon)$.

Similarly, we prove that if

$$
-\pi+2 \epsilon / \tau<t_{k_{0}}<2 \pi-2 \epsilon / \tau \text { for some } k_{0},
$$


then $(s, t) \in Y(\epsilon)$. If both (7.14) and (7.15) are violated, then $(s, t) \in Z(2 \epsilon)$ and so we obtain (7.13).

Next, we show that the integral over $Z(2 \epsilon)$ is asymptotically negligible. The set $Z(2 \epsilon)$ is a union of $2^{m+n-1}$ pairwise disjoint corners, where each corner is determined by a choice of the interval $[-\pi,-\pi+2 \epsilon / \tau]$ or $[\pi-2 \epsilon / \tau, \pi]$ for each coordinate $s_{j}$ and $t_{k}$. The transformation

$$
\begin{aligned}
& s_{j} \longmapsto\left\{\begin{array}{ll}
s_{j}+\pi & \text { if } s_{j} \in[-\pi,-\pi+2 \epsilon / \tau] \\
s_{j}-\pi & \text { if } s_{j} \in[\pi-2 \epsilon / \tau, \pi]
\end{array} \quad \text { for } \quad j=1, \ldots, m,\right. \\
& \text { and } \\
& t_{k} \longmapsto\left\{\begin{array}{ll}
t_{k}+\pi & \text { if } t_{k} \in[-\pi,-\pi+2 \epsilon / \tau] \\
t_{k}-\pi & \text { if } t_{k} \in[\pi-2 \epsilon / \tau, \pi]
\end{array} \quad \text { for } \quad k=1, \ldots, n-1\right.
\end{aligned}
$$

is measure-preserving and maps $Z(2 \epsilon)$ onto the cube

$$
I=\left\{(s, t): \quad\left|s_{j}\right|,\left|t_{k}\right| \leq 2 \epsilon / \tau \text { for all } j, k\right\} .
$$

In the product (7.4), it does not change the value of $f_{j k}$ except when $k=n$ (recall that $t_{n}=0$ on $\Pi_{0}$ ). Since $2 \epsilon / \tau<1$, by (7.5) the transformation increases the value of each function $f_{j n}$ by at least a factor of $\gamma(\delta)>1$. Therefore,

$$
\int_{Z(2 \epsilon)}|F(s, t)| d s d t \leq \exp \{-\Omega(m)\} \int_{I}|F(s, t)| d s d t
$$

and the proof follows by (7.13) and Proposition 7.3 .

Lemma 7.5. For $\epsilon>0$ let us define the cube

$$
I(\epsilon)=\left\{(s, t) \in \Pi_{0}: \quad\left|s_{j}\right|,\left|t_{k}\right| \leq \epsilon / \tau \quad \text { for all } \quad j, k\right\} .
$$

Then

$$
\int_{\Pi_{0} \backslash I(\epsilon)}|F(s, t)| d s d t \leq \exp \{-\gamma(\delta, \epsilon)(m+n)\} \int_{\Pi_{0}}|F(s, t)| d s d t
$$

for some $\gamma(\delta, \epsilon)>0$ and $m+n$ large enough.

Proof. Without loss of generality, we assume that $\epsilon<\delta$, so $\epsilon / \tau<1$.

Let $Y(\epsilon / 20)$ be the set of Lemma 7.4, so the integral of $|F(s, t)|$ over $\Pi \backslash Y(\epsilon / 20)$ is asymptotically negligible.

Let us choose a point $(s, t) \in Y(\epsilon / 20)$. We have

$$
l \epsilon / 20 \tau \leq s_{1} \leq(l+1) \epsilon / 20 \tau \quad \text { for some integer } l .
$$

Since $\left|s_{1}+t_{k}\right| \leq \epsilon / 20$, we obtain

$$
(-l-2) \epsilon / 20 \tau \leq t_{k} \leq(-l+1) \epsilon / 20 \tau \text { for } \quad k=1, \ldots, n-1
$$

and then similarly

$$
(l-2) \epsilon / 20 \tau \leq s_{j} \leq(l+3) \epsilon / 20 \tau \text { for } \quad j=1, \ldots, m .
$$

Let us denote

$$
w=(\underbrace{\epsilon / 20 \tau, \ldots, \epsilon / 20 \tau}_{m \text { times }} ; \underbrace{-\epsilon / 20 \tau, \ldots,-\epsilon / 20 \tau}_{n-1 \text { times }}, 0) .
$$


Hence we conclude that

$$
Y(\epsilon / 20) \subset \bigcup_{\substack{|l| \leq 1+20 \pi \tau / \epsilon \\ l \in \mathbb{Z}}} I(3 \epsilon / 20)+l w .
$$

Since $\tau$ is bounded by a polynomial in $m$ and $n$, the number of translates of the cube $I(3 \epsilon / 20)$ on the right-hand side of (17.16) is $(m+n)^{O(1)}$.

The translation

$$
(s, t) \longmapsto(s, t)+l w
$$

does not change the value of the functions $f_{j k}\left(s_{j}+t_{k}\right)$ in (7.4), unless $k=n$ (recall that $t_{n}=0$ on $\left.\Pi_{0}\right)$.

For $(s, t) \in I(3 \epsilon / 20)$ we have $\left|s_{j}\right| \leq 3 \epsilon / 20 \tau$ for all $j$. For $(s, t) \in I(3 \epsilon / 20)+l w$ with $|l| \geq 10$, we have $\left|s_{j}\right| \geq 7 \epsilon / 20 \tau$ for all $j$. Since $\epsilon / \tau<1$, for all $l$ in the union of (7.16) such that $|l| \geq 10$ and all $(s, t) \in I(3 \epsilon / 20)+l w$ we have $\rho\left(s_{j}\right) \geq 6 \epsilon / 20 \tau$ for all $j=1, \ldots, m$.

Using (7.4) and (7.5), we conclude that

$$
\begin{aligned}
|F(s, t)| \leq \exp \{-\Omega(m)\}|F(\tilde{s}, \tilde{t})| \text { for all }(\tilde{s}, \tilde{t}) \in I(3 \epsilon / 20) \\
\text { and for all }(s, t) \in I(3 \epsilon / 20)+l w \text { with }|l| \geq 10 .
\end{aligned}
$$

Since the number of translates in (17.16) is bounded by a polynomial in $(m+n)$ and since

$$
I(3 \epsilon / 20)+l w \subset I(\epsilon) \text { provided }|l| \leq 10,
$$

the proof follows by Lemma 7.4 .

To finish the proof of Theorem 7.1 we need a concentration inequality for strictly log-concave probability measures.

Theorem 7.6. Let $V$ be Euclidean space with the norm $\|\cdot\|$, let $B \subset V$ be a convex body, and let us consider a probability measure supported on $B$ with the density $e^{-U}$, where $u: B \longrightarrow \mathbb{R}$ is a function satisfying

$$
U(x)+U(y)-2 U\left(\frac{x+y}{2}\right) \geq c\|x-y\|^{2} \quad \text { for all } \quad x, y \in B
$$

and some constant $c>0$. For a point $x \in V$ and a closed subset $A \subset V$ we define the distance

$$
\operatorname{dist}(x, A)=\min _{y \in A}\|x-y\| .
$$

Let $A \subset B$ be a closed set such that $\mathbf{P}(A) \geq 1 / 2$. Then, for any $r \geq 0$ we have

$$
\mathbf{P}\{x \in B: \quad \operatorname{dist}(x, A) \geq r\} \leq 2 e^{-c r^{2}} .
$$

Proof. See, for example, Section 2.2 of [21] or Theorem 8.1 and its proof in [1, which, although stated for the Gaussian measure is adapted in a straightforward way to our situation.

Here is how we apply Theorem 7.6

Lemma 7.7. Let us choose $0<\epsilon \leq \delta^{2} / 10$. In the space $\mathbb{R}^{m+n}$ let us consider the hyperplane

$$
H=\left\{\left(s_{1}, \ldots, s_{m} ; t_{1}, \ldots, t_{n}\right): \quad \sum_{j=1}^{m} s_{j}=\sum_{k=1}^{n} t_{k}\right\} .
$$


Let $B \subset H$ be a convex body centrally symmetric around the origin: $(s, t) \in B$ if and only if $(-s,-t) \in B$, and such that for all $(s, t) \in B$ we have

$$
\begin{array}{ll}
\left|s_{j}\right| \leq \epsilon / \tau & \text { for } \quad j=1, \ldots, m, \\
\left|t_{k}\right| \leq \epsilon / \tau & \text { for } \quad k=1, \ldots, n .
\end{array}
$$

Let us consider the probability measure on $B$ with the density proportional to $|F(s, t)|$. Then, for any $\kappa>0$ we have

$$
\mathbf{P}\left\{(s, t) \in B: \quad\left|s_{j}\right|,\left|t_{k}\right| \leq \frac{\ln (m+n)}{2 \tau \sqrt{m+n}} \quad \text { for all } j, k\right\} \geq 1-(m+n)^{-\kappa},
$$

provided $m+n \geq \gamma(\delta, \kappa)$ for some constant $\gamma(\delta, \kappa)>0$.

Proof. Let $f_{j k}$ be the functions defined in Section 7.2 and let

$$
u_{j k}=-\ln f_{j k} .
$$

We define

$$
U(s, t)=a+\sum_{\substack{1 \leq j \leq m \\ 1 \leq k \leq n}} u_{j k}\left(s_{j}+t_{k}\right) \quad \text { for } \quad(s, t) \in B,
$$

where $a$ is a constant chosen in such a way that

$$
e^{-U}=e^{-a}|F(s, t)|
$$

is a probability density on $B$. It follows by (7.6) that

$$
\begin{gathered}
u_{j k}(x)+u_{j k}(y)-2 u_{j k}\left(\frac{x+y}{2}\right) \geq \Omega\left(\tau^{2}(x-y)^{2}\right) \\
\text { provided }|x|,|y| \leq 2 \epsilon / \tau .
\end{gathered}
$$

Let us consider the map

$$
M:\left(s_{1}, \ldots, s_{m} ; t_{1}, \ldots, t_{n}\right) \longmapsto\left(\ldots s_{j}+t_{k} \ldots\right)
$$

as a map $M: H \longmapsto \mathbb{R}^{m n}$. From Lemma 3.5

$$
\|M x\|^{2} \geq \min \{m, n\}\|x\|^{2} \text { for all } x \in H,
$$

where $\|\cdot\|$ is the Euclidean norm in the corresponding space. It follows then by (17.17) that

$$
U(x)+U(y)-2 U\left(\frac{x+y}{2}\right) \geq \Omega\left(\tau^{2} n\|x-y\|^{2}\right) \quad \text { for all } \quad x, y \in B .
$$

Now we apply Theorem 7.6 with

$$
c=\Omega\left(\tau^{2} n\right)
$$

to the probability density $e^{-U}$ on $B$.

For $j=1, \ldots, m$, let $S_{j}^{+}$be the set consisting of the points $(s, t) \in B$ with $s_{j} \geq 0$, let $S_{j}^{-}$be the set consisting of the points $(s, t) \in B$ with $s_{j} \leq 0$, let $T_{k}^{+}$be the set consisting of the points $(s, t) \in B$ with $t_{k} \geq 0$ and let $T_{k}^{-}$be the set consisting of the points $(s, t) \in B$ with $t_{k} \leq 0$. Since both $B$ and the probability measure are invariant under the symmetry

$$
(s, t) \longmapsto(-s,-t),
$$

we have

$$
\mathbf{P}\left(S_{j}^{+}\right)=\mathbf{P}\left(S_{j}^{-}\right)=\mathbf{P}\left(T_{k}^{+}\right)=\mathbf{P}\left(T_{k}^{-}\right)=\frac{1}{2} .
$$


We note that

$$
\begin{aligned}
& \text { if } \quad\left|s_{j}\right| \geq r, \quad \text { then } \operatorname{dist}\left((s, t), S_{j}^{+}\right), \operatorname{dist}\left((s, t), S_{j}^{-}\right) \geq r \quad \text { and } \\
& \text { if } \quad\left|t_{k}\right| \geq r, \quad \text { then } \operatorname{dist}\left((s, t), T_{k}^{+}\right), \operatorname{dist}\left((s, t), T_{k}^{-}\right) \geq r .
\end{aligned}
$$

Applying Theorem 7.6 with

$$
r=\frac{\ln (m+n)}{2 \tau \sqrt{m+n}}
$$

we conclude that for all $j$ and $k$

$$
\begin{aligned}
& \mathbf{P}\left\{(s, t) \in B: \quad\left|s_{j}\right|>\frac{\ln (m+n)}{2 \tau \sqrt{m+n}}\right\} \leq \exp \left\{-\Omega\left(\ln ^{2} m\right)\right\} \quad \text { and } \\
& \mathbf{P}\left\{(s, t) \in B: \quad\left|t_{k}\right|>\frac{\ln (m+n)}{2 \tau \sqrt{m+n}}\right\} \leq \exp \left\{-\Omega\left(\ln ^{2} n\right)\right\}
\end{aligned}
$$

and the proof follows.

Now we are ready to prove Theorem 7.1 .

7.3. Proof of Theorem 7.1. Let us choose an $0<\epsilon \leq \delta^{2} / 10$ as in Lemma 7.7 and let $H \subset \mathbb{R}^{m+n}$ be the hyperplane defined in Lemma 7.7. We identify $\mathbb{R}^{m+n-1}$ with the hyperplane $\tau_{n}=0$ in $\mathbb{R}^{m+n}$. We consider a linear transformation $T: H \longrightarrow \mathbb{R}^{m+n-1}$,

$$
\left(s_{1}, \ldots, s_{m} ; t_{1}, \ldots, t_{n}\right) \longmapsto\left(s_{1}+t_{n}, \ldots, s_{m}+t_{n} ; t_{1}-t_{n}, \ldots, t_{n-1}-t_{n}, 0\right) .
$$

The inverse linear transformation

$$
T^{-1}: \quad\left(s_{1}^{\prime}, \ldots, s_{m}^{\prime} ; t_{1}^{\prime}, \ldots, t_{n-1}^{\prime}, 0\right) \longmapsto\left(s_{1}, \ldots, s_{m} ; t_{1}, \ldots, t_{n}\right)
$$

is computed as follows:

$$
t_{n}=\frac{s_{1}^{\prime}+\ldots+s_{m}^{\prime}-t_{1}^{\prime}-\ldots-t_{n-1}^{\prime}}{m+n}, \quad s_{j}=s_{j}^{\prime}-t_{n}, \quad t_{k}=t_{k}^{\prime}+t_{n} .
$$

Let us consider the cube $I=I(\epsilon / 2) \subset \mathbb{R}^{m+n-1}$ defined by the inequalities

$$
\left|s_{j}\right|,\left|t_{k}\right| \leq \epsilon / 2 \tau \quad \text { for } \quad j=1, \ldots, m \text { and } k=1, \ldots, n-1 .
$$

By Lemma 7.5 we have

$$
\int_{\Pi_{0} \backslash I}|F(s, t)| d s d t \leq \exp \{-\Omega(m+n)\} \int_{\Pi_{0}}|F(s, t)| d s d t
$$

for all sufficiently large $m$ and $n$.

Let $B=T^{-1}(I) \subset H$. Then $B$ is centrally symmetric and convex, and for all $(s, t) \in B$ we have $\left|s_{j}\right|,\left|t_{k}\right| \leq \epsilon / \tau$ for all $j$ and $k$. Let

$$
\begin{array}{cl}
A=\{(s, t) \in B: & \left|s_{j}\right| \leq \frac{\ln (m+n)}{2 \tau \sqrt{m+n}} \quad \text { for } \quad j=1, \ldots, m \quad \text { and } \\
\left.\left|t_{k}\right| \leq \frac{\ln (m+n)}{2 \tau \sqrt{m+n}} \quad \text { for } \quad k=1, \ldots, n\right\} .
\end{array}
$$

By Lemma 7.7,

$$
\int_{B \backslash A}|F(s, t)| d s d t \leq(m+n)^{-\kappa} \int_{B}|F(s, t)| d s d t
$$

provided $m+n \geq \gamma(\delta, \kappa)$ for some $\gamma(\delta, \kappa)>0$. Now, the push-forward of the probability measure on $B$ with the density proportional to $|F(s, t)|$ under the map $T$ 
is the probability measure on $I$ with the density proportional to $|F(s, t)|$. Moreover, the image $T(A)$ lies in the cube $\mathcal{U}$ defined by the inequalities

$$
\left|s_{j}\right|\left|t_{k}\right| \leq \frac{\ln (m+n)}{\tau \sqrt{m+n}} \quad \text { for } \quad j=1, \ldots, m \quad \text { and } \quad k=1, \ldots, n-1 .
$$

Therefore,

$$
\int_{I \backslash \mathcal{U}}\left|F(s, t) d s d t \leq(m+n)^{-\kappa} \int_{I}\right| F(s, t) \mid d s d t
$$

provided $m+n \geq \gamma(\delta, \kappa)$ for some $\gamma(\delta, \kappa)>0$. The proof now follows by (7.18) and (7.19).

\section{Proof of Theorem 1.1}

First, we prove Theorem 1.1 assuming, additionally, that $\tau \leq(m+n)^{1 / \delta}$ in (1.3).

8.1. Proof of Theorem 1.1 under the additional assumption that $\tau$ is bounded by a polynomial in $m+n$. All constants implicit in the " $O$ " and " $\Omega$ " notation below may depend only on the parameter $\delta$. We say that $m$ and $n$ are sufficiently large provided $m+n \geq \gamma(\delta)$ for some constant $\gamma(\delta)>0$.

As in Corollary 2.2. we represent the number $\#(R, C)$ of tables as the integral

$$
\#(R, C)=\frac{e^{g(Z)}}{(2 \pi)^{m+n-1}} \int_{\Pi_{0}} F(s, t) d s d t .
$$

Let $\mathcal{U} \subset \Pi_{0}$ be the neighborhood of the origin as defined in Theorems 6.1 and 7.1 From parts (2), (3) and (4) of Theorem 6.1 we conclude that the integrals of $F(s, t)$ and $|F(s, t)|$ over $\mathcal{U}$ are of the same order, that is

$$
\int_{\mathcal{U}}|F(s, t)| d s d t \leq O\left(\left|\int_{\mathcal{U}} F(s, t) d s d t\right|\right)
$$

provided $m+n$ is sufficiently large. Theorem 7.1 implies then that the integral of $F(s, t)$ over $\Pi_{0} \backslash \mathcal{U}$ is asymptotically negligible: for any $\kappa>0$ we have

$$
\left|\int_{\Pi_{0} \backslash \mathcal{U}} F(s, t) d s d t\right| \leq(m+n)^{-\kappa}\left|\int_{\mathcal{U}} F(s, t) d s d t\right|
$$

provided $m+n>\gamma(\delta, \kappa)$ for some $\gamma(\delta, \kappa)>0$.

We use part (3) of Theorem 6.1 to compute

$$
\int_{\mathcal{U}} F(s, t) d s d t
$$

Identifying $\mathbb{R}^{m+n-1}$ with the hyperplane $\tau_{n}=0$ in $\mathbb{R}^{m+n}$, we note that

$$
\Xi=\int_{\mathbb{R}^{m+n-1}} e^{-q} d s d t=\frac{\pi^{(m+n-1) / 2}}{\sqrt{\operatorname{det} q \mid \mathbb{R}^{m+n-1}}},
$$

and that by Lemma 3.4 we have

$$
\operatorname{det} q\left|\mathbb{R}^{m+n-1}=\frac{1}{m+n} \operatorname{det} q\right| H,
$$

where $H$ is the hyperplane orthogonal to the null-space of $q$. 
To conclude the proof, we note that by Lemma 3.1 the values of

$$
\mu=\mathbf{E} f^{2} \text { and } \quad \nu=\mathbf{E} h
$$

can be computed with respect to the Gaussian probability measure with the density proportional to $e^{-q}$ in an arbitrary hyperplane $L \subset \mathbb{R}^{m+n}$ not containing the nullspace of $q$.

To handle the case of super-polynomial $\tau$, we use a result of 18 (see Lemma 3 there) which shows that $\#(R, C) \approx \operatorname{vol} P(R, C)$ provided the margins $R$ and $C$ are large enough (it suffices to have $\left.\tau \geq(m n)^{2}\right)$. Then we note that

$$
\operatorname{vol} P(\alpha R, \alpha C)=\alpha^{(m-1)(n-1)} \operatorname{vol} P(R, C) \text { for } \quad \alpha>0
$$

and show that the formula of Theorem 1.1 scales similarly. In the next three lemmas we show that the typical matrix of $(\alpha R, \alpha C)$ is approximately the typical matrix of $(R, C)$ multiplied by $\alpha$ and that the typical matrix of $(R, C)$ is approximately the typical matrix of $\left(R^{\prime}, C^{\prime}\right)$ if $R^{\prime} \approx R$ and $C^{\prime} \approx C$. We then complete our proof of Theorem 1.1.

In Lemmas 8.1 and 8.2 below, all implicit constants in the " $O$ " notation are absolute.

Lemma 8.1. Let $R=\left(r_{1}, \ldots, r_{m}\right)$ and $C=\left(c_{1}, \ldots, c_{n}\right)$ be positive (not necessarily integer) vectors such that $r_{1}+\ldots+r_{m}=c_{1}+\ldots+c_{n}$ and let $Z=\left(\zeta_{j k}\right)$ be the typical matrix maximizing the the value of

$$
g(X)=\sum_{\substack{1 \leq j \leq m \\ 1 \leq k \leq n}}\left(\left(x_{j k}+1\right) \ln \left(x_{j k}+1\right)-x_{j k} \ln x_{j k}\right)
$$

on the polytope $P(R, C)$ of $m \times n$ non-negative matrices with row sums $R$ and column sums $C$.

Let

$$
\begin{aligned}
& r_{-}=\min _{j=1, \ldots, m} r_{j}, \quad c_{-}=\min _{k=1, \ldots, n} c_{k} \quad \text { and } \\
& r_{+}=\max _{j=1, \ldots, m} r_{j}, \quad c_{+}=\max _{k=1, \ldots, n} c_{k} .
\end{aligned}
$$

Then

$$
\zeta_{j k} \geq \frac{r_{-} c_{-}}{r_{+} m} \quad \text { and } \quad \zeta_{j k} \geq \frac{c_{-} r_{-}}{c_{+} n} \text { for all } j, k .
$$

Proof. This is part (1) of Theorem 3.3 of [7.

Lemma 8.2. Let $Z=\left(\zeta_{j k}\right)$ be the $m \times n$ typical matrix of margins $(R, C)$ such that

$$
\delta \tau \leq \zeta_{j k} \leq \tau \text { for all } j, k,
$$

for some $0<\delta<1 / 2$ and some $\tau>1$.

Let $0<\alpha<1$ and let $X=\left(\xi_{j k}\right)$ be the typical matrix of margins $(\alpha R, \alpha C)$.

Then the following holds:

(1) We have

$$
\xi_{j k} \geq \alpha \delta^{2} \tau \text { for all } j, k
$$

(2) Suppose $\alpha \delta^{2} \tau>1$. Then

$$
|g(Z)+m n \ln \alpha-g(X)|=O\left(\frac{m n}{\alpha \delta^{2} \tau}\right) .
$$


(3) There exists an absolute constant $\gamma>1$ such that if $\alpha \delta^{4} \tau \geq \gamma m n$, then

$$
\left|\xi_{j k}-\alpha \zeta_{j k}\right| \leq \epsilon \alpha \zeta_{j k} \text { for all } j, k
$$

and

$$
\epsilon=O\left(\sqrt{\frac{m n}{\alpha \delta^{4} \tau}}\right) .
$$

Proof. Let $R=\left(r_{1}, \ldots, r_{m}\right)$ and $C=\left(c_{1}, \ldots, c_{n}\right)$. Thus in Lemma 8.1 we have

$$
r_{-} \geq \delta n \tau, \quad c_{-} \geq \delta m \tau \text { and } r_{+} \leq \tau n
$$

Applying Lemma 8.1 to the scaled margins $(\alpha R, \alpha C)$, we obtain part (1).

Since $\alpha Z \in P(\alpha R, \alpha C)$ and $\alpha^{-1} X \in P(R, C)$, we have

$$
g(Z) \geq g\left(\alpha^{-1} X\right) \text { and } g(X) \geq g(\alpha Z) .
$$

Since for $x \geq 1$ we have

$$
\begin{aligned}
g(x) & =(x+1) \ln (x+1)-x \ln x+(x+1) \ln x-(x+1) \ln x \\
& =(x+1) \ln \left(1+\frac{1}{x}\right)+\ln x=1+\ln x+O\left(\frac{1}{x}\right),
\end{aligned}
$$

from (8.1) we obtain part (2).

Let us consider the interval $[X, \alpha Z] \subset P(\alpha R, \alpha C)$. Since $g$ is concave, we have

$$
|g(Y)-g(X)|=O\left(\frac{m n}{\alpha \delta^{2} \tau}\right) \quad \text { for all } \quad Y \in[X, \alpha Z] .
$$

Suppose that for some $0<\epsilon<1 / 2$ we have

$$
\left|\xi_{j k}-\alpha \zeta_{j k}\right|>\epsilon \alpha \zeta_{j k} \quad \text { for some } j, k .
$$

Then there is a matrix $Y \in[X, \alpha Z], Y=\left(\eta_{j k}\right)$, such that $\left|\eta_{j k}-\alpha \zeta_{j k}\right|=\epsilon \alpha \zeta_{j k}$. We note that

$$
g^{\prime \prime}(x)=-\frac{1}{x(x+1)}
$$

and, in particular,

$$
g^{\prime \prime}(x) \leq-\frac{1}{8 \alpha^{2} \tau^{2}} \quad \text { for all } \quad x \in\left[\eta_{j k}, \alpha \zeta_{j k}\right]
$$

Next, we are going to exploit the strong concavity of $g$ and use the following standard inequality: If $g^{\prime \prime}(x) \leq-\beta$ for some $\beta>0$ and all $a \leq x \leq b$, then

$$
g\left(\frac{a+b}{2}\right)-\frac{1}{2} g(a)-\frac{1}{2} g(b) \geq \frac{\beta(b-a)^{2}}{8} .
$$

Applying the above inequality to $g$ with $a=\eta_{j k}, b=\alpha \zeta_{j k}$ and $\beta=1 / 8 \alpha^{2} \tau^{2}$, we obtain

$$
g\left(\frac{\eta_{j k}+\alpha \zeta_{j k}}{2}\right)-\frac{1}{2} g\left(\eta_{j k}\right)-\frac{1}{2} g\left(\alpha \zeta_{j k}\right) \geq \frac{\epsilon^{2} \delta^{2}}{64} .
$$

Let $W=(Y+\alpha Z) / 2$. Then $W \in[X, \alpha Z]$ and by (8.3)

$$
g(W) \geq g(X)+\frac{\epsilon^{2} \delta^{2}}{64}-O\left(\frac{m n}{\alpha \delta^{2} \tau}\right) .
$$

Since $g(W) \leq g(X)$, the proof follows. 
Lemma 8.3. Let $Z=\left(\zeta_{j k}\right)$ be the $m \times n$ typical matrix of margins $R=\left(r_{1}, \ldots, r_{m}\right)$ and $C=\left(c_{1}, \ldots, c_{n}\right)$ such that

$$
\delta \tau \leq \zeta_{j k} \leq \tau \text { for all } j, k,
$$

for some $0<\delta<1 / 2$ and some $\tau>1$.

Let $0<\epsilon<1 / 2$ and let $X=\left(\xi_{j k}\right)$ be the typical matrix of some margins $R=\left(r_{1}^{\prime}, \ldots, r_{m}^{\prime}\right)$ and $C^{\prime}=\left(c_{1}^{\prime}, \ldots, c_{n}^{\prime}\right)$ such that

$$
\begin{aligned}
& (1-\epsilon) r_{j} \leq r_{j}^{\prime} \leq r_{j} \text { for } j=1, \ldots, m \quad \text { and } \\
& (1-\epsilon) c_{k} \leq c_{k}^{\prime} \leq c_{k} \text { for } k=1, \ldots, n .
\end{aligned}
$$

Suppose that $\delta^{2} \tau>1$. Then the following holds:

(1) We have

$$
|g(X)-g(Z)|=O(m n \epsilon) .
$$

(2) There exists an absolute constant $\gamma$ such that if $\epsilon \leq \gamma \delta^{2} / m n$, then

$$
\left|\xi_{j k}-\zeta_{j k}\right| \leq \beta \zeta_{j k} \text { for all } j, k
$$

and

$$
\beta=O\left(\sqrt{\frac{m n \epsilon}{\delta^{2}}}\right)
$$

Proof. Suppose that $A=\left(a_{1}, \ldots, a_{m}\right)$ and $B=\left(b_{1}, \ldots, b_{n}\right)$ are margins and $A^{\prime}=$ $\left(a_{1}^{\prime}, \ldots, a_{m}^{\prime}\right)$ and $B^{\prime}=\left(b_{1}^{\prime}, \ldots, b_{n}^{\prime}\right)$ are some other margins such that $a_{j}^{\prime} \leq a_{j}$ and $b_{k}^{\prime} \leq b_{k}$ for all $j$ and $k$. Then there exists a non-negative matrix $D$ with margins $a_{j}-a_{j}^{\prime}$ and $b_{k}-b_{k}^{\prime}$ and for such a $D$ we have $Y+D \subset P(A, B)$ for all $Y \in P\left(A^{\prime}, C^{\prime}\right)$. Snce $g$ is monotone increasing, we obtain

$$
\begin{aligned}
g((1-\epsilon) Z) & \leq \max _{Y \in(1-\epsilon) P(R, C)} g(Y) \leq g(X)=\max _{Y \in P\left(R^{\prime}, C^{\prime}\right)} g(Y) \\
& \leq \max _{Y \in P(R, C)} g(Y)=g(Z) .
\end{aligned}
$$

Hence

$$
g((1-\epsilon) Z) \leq g(X) \leq g(Z)
$$

and from (8.2) we deduce part (2).

We note that $Z$ is the maximum point of $g$ on the polytope of non-negative $m \times n$ matrices with the row sums not exceeding $R$ and column sums not exceeding $C$. Therefore,

$$
|g(Y)-g(Z)|=O(m n \epsilon) \quad \text { for all } \quad Y \in[X, Z] .
$$

Suppose that for some $0<\beta<1 / 2$ we have

$$
\left|\xi_{j k}-\zeta_{j k}\right|>\beta \zeta_{j k} \text { for some } j, k \text {. }
$$

Then there is a matrix $Y \in[X, Z], Y=\left(\eta_{j k}\right)$, such that $\left|\eta_{j k}-\zeta_{j k}\right|=\beta \zeta_{j k}$. As in the proof of Lemma 8.2 , we argue that

$$
g^{\prime \prime}(x) \leq-\frac{1}{8 \tau^{2}} \quad \text { for all } \quad x \in\left[\eta_{j k}, \zeta_{j k}\right]
$$

and that

$$
g\left(\frac{\eta_{j k}+\zeta_{j k}}{2}\right)-\frac{1}{2} g\left(\eta_{j k}\right)-\frac{1}{2} g\left(\zeta_{j k}\right) \geq \frac{\beta^{2} \delta^{2}}{64} .
$$


Let $W=(Y+Z) / 2$. Then $W \in[Y, Z]$, and by (8.4)

$$
g(W) \geq g(Z)+\frac{\beta^{2} \delta^{2}}{64}-O(m n \epsilon) .
$$

Since $g(W) \leq g(Z)$, the proof follows.

8.2. Proof of Theorem 1.1, All implicit constants in the " $O$ " and " $\Omega$ " notation below may depend on parameter $\delta$ only.

In view of Section 8.1, without loss of generality we assume that $\tau \geq(m+n)^{10}$ in (1.3). As follows by [18, as long as $\tau \geq(m+n)^{2}$ we have

$$
\#(R, C)=\operatorname{vol} P(R, C)\left(1+O\left(\frac{1}{m+n}\right)\right),
$$

where vol $P(R, C)$ is the volume of the polytope of the set of $m \times n$ non-negative matrices with row sums $R$ and column sums $C$ normalized in such a way that the volume of the fundamental domain of the $(m-1)(n-1)$-dimensional lattice consisting of the $m \times n$ integer matrices with zero row and column sums is equal to 1.

Let $\alpha=(m+n)^{9} \tau^{-1}$ and let

$$
\hat{R}=\left(\hat{r}_{1}, \ldots, \hat{r}_{m}\right) \quad \text { and } \quad \hat{C}=\left(\hat{c}_{1}, \ldots, \hat{c}_{n}\right)
$$

be positive integer margins (so $\hat{r}_{1}+\ldots+\hat{r}_{m}=\hat{c}_{1}+\ldots+\hat{c}_{n}$ ) such that

$$
\begin{gathered}
(1-\epsilon) \alpha r_{j} \leq \hat{r}_{j} \leq \alpha r_{j} \text { and }(1-\epsilon) \alpha c_{k} \leq \hat{c}_{k} \leq \alpha c_{k} \\
\text { for some } 0<\epsilon<(m+n)^{-7} .
\end{gathered}
$$

Then

$$
\begin{aligned}
\#(R, C) & \approx \operatorname{vol} P(R, C) \approx \alpha^{(m-1)(1-n)} \operatorname{vol} P(\hat{R}, \hat{C}) \\
& \approx \alpha^{(m-1)(1-n)} \#(\hat{R}, \hat{C}),
\end{aligned}
$$

where " $\approx$ " denotes the equality up to a $O\left((m+n)^{-1}\right)$ relative error.

Let $Z=\left(\zeta_{j k}\right)$ be the typical matrix of margins $(R, C)$ and let $\hat{Z}=\left(\hat{\zeta}_{j k}\right)$ be the typical matrix of margins $(\hat{R}, \hat{C})$. By Lemmas 8.2 and 8.3 , we have

$$
\begin{aligned}
& |g(Z)+m n \ln \alpha-g(\hat{Z})|=O\left(\frac{1}{(m+n)^{5}}\right) \text { and } \\
& \left|\hat{\zeta}_{j k}-\alpha \zeta_{j k}\right|=O\left(\frac{\alpha \zeta_{j k}}{(m+n)^{2}}\right) \text { for all } j, k .
\end{aligned}
$$

Let $q, \hat{q}: \mathbb{R}^{m+n} \longrightarrow \mathbb{R}$ be the quadratic forms associated by (1.5) with margins $(R, C)$ and $(\hat{R}, \hat{C})$, respectively. Then by the second estimate of (8.6) it follows that

$$
\hat{q}(s, t) \approx \alpha^{2} q(s, t) \text { for all }(s, t) \in \mathbb{R}^{m+n},
$$

where " $\approx$ " stands for the equality up to a $O\left((m+n)^{-2}\right)$ relative error. It follows then by the first estimate of (8.6) that the Gaussian term (1.7) for margins $(R, C)$, up to a relative error of $O\left((m+n)^{-1}\right)$, is obtained by multiplying the Gaussian term for margins $(\hat{R}, \hat{C})$ by $\alpha^{(m-1)(1-n)}$.

Similarly, we show that the Edgeworth correction factor (1.8) changes negligibly as we pass from $(R, C)$ to $(\hat{R}, \hat{C})$. By making substitutions

$$
(s, t) \longmapsto \tau^{-1}(s, t) \text { and }(s, t) \longmapsto \alpha^{-1} \tau^{-1}(s, t),
$$


respectively, we express the quantities $(\mu, \nu)$ for margins $(R, C)$ and $(\hat{\mu}, \hat{\nu})$ for margins $(\hat{R}, \hat{C})$ as

$$
\mu=\mathbf{E} f^{2}, \nu=\mathbf{E} h \quad \text { and } \quad \hat{\mu}=\mathbf{E} \hat{f}^{2}, \hat{\nu}=\mathbf{E} \hat{h},
$$

where the expectations $\mu$ and $\nu$ are taken with respect to the Gaussian measure on $H$ with the density proportional to $e^{-\psi}$ and the expectations $\hat{\mu}$ and $\hat{\nu}$ are taken with respect to the Gaussian measure with the density proportional to $e^{-\hat{\psi}}$, where $\psi$ and $\hat{\psi}$ are positive definite quadratic forms within a relative error of $O\left((m+n)^{-2}\right)$ of each other. Moreover, $f^{2}$ and $\hat{f}^{2}$ are homogeneous polynomials of degree 6 and $h$ and $\hat{h}$ are homogeneous polynomials of degree 4 such that

$$
\begin{aligned}
& f^{2}(s, t), \hat{f}^{2}(s, t)=O\left(\sum_{j, k}\left|s_{j}+t_{k}\right|^{3}\right)^{2}, \\
& h(s, t), \hat{h}(s, t)=O\left(\sum_{j, k}\left(s_{j}+t_{k}\right)^{4}\right) \text { and } \\
& \left|f^{2}(s, t)-\hat{f}^{2}(s, t)\right|=O\left(\frac{1}{(m+n)^{2}}\right)\left(\sum_{j, k}\left|s_{j}+t_{k}\right|^{3}\right)^{2}, \\
& |h(s, t)-\hat{h}(s, t)|=O\left(\frac{1}{(m+n)^{2}}\right) \sum_{j, k}\left(s_{j}+t_{k}\right)^{4} .
\end{aligned}
$$

Since by Lemma 3.5. the minimum eigenvalues of $\psi$ and $\hat{\psi}$ are $\Omega(m+n)$, standard estimates imply that $\exp \{-\mu / 2+\nu\}$ approximates $\exp \{-\hat{\mu} / 2+\hat{\nu}\}$ within a $O\left((m+n)^{-1}\right)$ relative error.

We have

$$
\hat{\zeta}_{j k}=O\left((m+n)^{9}\right) \quad \text { for all } j, k,
$$

and hence by the result of Section 8.1 we can apply Theorem 1.1 to estimate $\#(\hat{R}, \hat{C})$. The proof then follows by (8.5).

\section{REFERENCES}

1. K. Ball, An elementary introduction to modern convex geometry, in Flavors of Geometry, Mathematical Sciences Research Institute Publications, vol. 31, Cambridge University Press, Cambridge, 1997, pp. 1-58. MR.1491097 (99f:52002)

2. A. Barvinok, Computing mixed discriminants, mixed volumes, and permanents, Discrete \& Computational Geometry 18 (1997), 205-237. MR:1455515 (98m:52011)

3. A. Barvinok, Asymptotic estimates for the number of contingency tables, integer flows, and volumes of transportation polytopes, International Mathematics Research Notices 2009 (2009), 348-385. MR2482118 (2010c:52019)

4. A. Barvinok, What does a random contingency table look like?, Combinatorics, Probability and Computing 19 (2010), 517-539. MR.2647491

5. A. Barvinok and J.A. Hartigan, Maximum entropy Gaussian approximation for the number of integer points and volumes of polytopes, Advances in Applied Mathematics 45 (2010), 252-289. MR2646125(2011d:52026)

6. A. Barvinok and J.A. Hartigan, The number of graphs and a random graph with a given degree sequence, Random Structures \& Algorithms, to appear.

7. A. Barvinok, Z. Luria, A. Samorodnitsky and A. Yong, An approximation algorithm for counting contingency tables, Random Structures \& Algorithms 37 (2010), 25-66. MR2674620 
8. A. Békéssy, P. Békéssy, and J. Komlós, Asymptotic enumeration of regular matrices, Studia Scientiarum Mathematicarum Hungarica, 7 (1972), 343-353. MR0335342 (49:124)

9. E. Bender, The asymptotic number of non-negative integer matrices with given row and column sums, Discrete Mathematics 10 (1974), 217-223. MR.0389621(52:10452)

10. E. R. Canfield and B. D. McKay Asymptotic enumeration of integer matrices with large equal row and column sums, Combinatorica 30 (2010), no. 6, 655-680. MR2789732 (2012b:05029)

11. Y. Chen, P. Diaconis, S.P.Holmes, and J.S. Liu, Sequential Monte Carlo methods for statistical analysis of tables, Journal of the American Statistical Association 100 (2005), 109-120. MR 2156822(2006f:62062)

12. M. Cryan and M. Dyer, A polynomial-time algorithm to approximately count contingency tables when the number of rows is constant, Special issue of STOC 2002 (Montreal, QC), Journal of Computer and System Sciences 67 (2003), 291-310. MR2022833 (2005h:68177)

13. J.A. De Loera, Counting and estimating lattice points: tools from algebra, analysis, convexity, and probability, Optima 81(2009), 1-9.

14. J.A. De Loera, Appendix: details on experiments (counting and estimating lattice points), Optima 81(2009), 17-22.

15. P. Diaconis and B. Efron, Testing for independence in a two-way table: new interpretations of the chi-square statistic. With discussions and with a reply by the authors, The Annals of Statistics 13 (1985), 845-913. MR803747 (87c:62106)

16. P. Diaconis and A. Gangolli, Rectangular arrays with fixed margins, in Discrete Probability and Algorithms (Minneapolis, MN, 1993), The IMA Volumes in Mathematics and its Applications, vol. 72, Springer, New York, 1995, pp. 15-41. MR.1380519 (97e:05013)

17. J. Duoandikoetxea, Reverse Hölder inequalities for spherical harmonics, Proceedings of the American Mathematical Society 101 (1987), 487-491. MR908654 (88m:42040)

18. M. Dyer, R. Kannan, and J. Mount, Sampling contingency tables, Random Structures \& Algorithms 10 (1997), 487-506. MR1608222 (98i:68124)

19. C. Greenhill and B.D. McKay, Asymptotic enumeration of sparse nonnegative integer matrices with specified row and column sums, Advances in Applied Mathematics 41 (2008), 459-481. MR 2459445 (2009i:05021)

20. I.J. Good and J.F.Crook, The enumeration of arrays and a generalization related to contingency tables, Discrete Math. 19 (1977), 23-45. MR0541011 (58:27527)

21. M. Ledoux, The Concentration of Measure Phenomenon Mathematical Surveys and Monographs, vol. 89, American Mathematical Society, Providence, RI, 2001. MR1849347 (2003k:28019)

22. Z. Luria, Counting contingency tables with balanced margins, manuscript, 2008.

23. B.J. Morris, Improved bounds for sampling contingency tables, Random Structures \& Algorithms 21 (2002), 135-146. MR1917354 (2003d:62141)

24. Yu. Nesterov and A. Nemirovskii, Interior-Point Polynomial Algorithms in Convex Programming, SIAM Studies in Applied Mathematics, vol. 13, Society for Industrial and Applied Mathematics (SIAM), Philadelphia, PA, 1994. MR1258086 (94m:90005)

25. V. Zipunnikov, J.G. Booth and R. Yoshida, Counting tables using the double saddlepoint approximation, Journal of Computational and Graphical Statistics 18 (2009), 915-929.

26. A. Zvonkin, Matrix integrals and map enumeration: an accessible introduction, in Combinatorics and physics (Marseilles, 1995), Mathematical and Computer Modelling 26 (1997), 281-304. MR1492512 (98m:81037)

Department of Mathematics, University of Michigan, Ann Arbor, Michigan 481091043

E-mail address: barvinok@umich.edu

Department of Statistics, Yale University, New Haven, Connecticut 06520-8290

E-mail address: john.hartigan@yale.edu 